\title{
The Implications of Alternative Saving and Expectations Hypotheses for Choices of Technique and Patterns of Growth
}

David Cass and Joseph E. Stiglitz

Yale University

\section{Introduction}

It has become increasingly clear that there are serious problems with the concept of aggregate capital. It is now known, for instance, that, if there are many vintages of machines, the output of the economy can be described by a simple neoclassical production function with an aggregate capital stock if and only if technical change is purely capital augmenting (Fisher, 1965); that in steady states consumption per capita may not be a monotonic function of the rate of interest, even for rates of interest greater than the rate of growth; and that the value of the capital stock per man (using any price system) may not be monotonic in $r$ (Wicksell effects) (Champernowne, 1953-54). The heterogeneity of capital goods, moreover, raises new problems for the dynamic behavior of the economy. Investment must be allocated, and to do this firms must form expectations of future prices, wages, and interest rates.

The purpose of this paper is to investigate in detail the dynamics of a simple model with heterogeneous capital goods under alternative assumptions about expectations formation and saving. Before investment takes place, the entrepreneur has a choice over a large number (to be precise, a continuum) of types of machines; those which require more resources today require less labor per unit of output in the future. But once the machine has been constructed, it cannot be altered. This is just the exante smooth substitutability-ex post rigid complementarity model (or the putty-clay model), alternative versions of which have been studied by Johansen (1959) and Solow (1962). (See also Phelps, 1963; Akerlof, 1967; Sheshinski, 1967; and Stiglitz, 1968.) This model raises, moreover, the interesting problem of economic obsolescence: machines may be con-

The research described in this paper was carried out under a grant from the National Science Foundation. 
structed which subsequently, because of higher wage rates, are no longer profitable to operate.

We shall be interested in determining how the dynamic behavior of this economy differs from that of the economy with malleable capital, as analyzed by Solow (1956) and Swan (1956) for the descriptive growth model and by Ramsey (1928) for the optimal growth model. It will be shown that, although there are fundamental differences in short-run behavior, in the long run the economy evolves much like the economy described by the simpler malleable capital models. On the other hand, econometric estimation based on the use of the malleable capital model, such as that of Solow (1957) in estimating the residual, or Arrow, Chenery, Minhas, and Solow (1961) in estimating the elasticity of substitution, may encounter serious biases from the specification error.

In Part II we set forth the basic model; Part III analyzes the behavior of the economy under static expectations and alternative saving assumptions. Part IV presents the perfect foresight model with a constant saving rate; in Part V the saving rate is chosen to maximize the discounted sum of consumption. Part VI sketches a model in which firms act on probability distributions of possible wages rather than on point estimates. In Part VII the implications of the model for econometric estimation are outlined.

\section{The Model}

The technology underlying our model is perhaps the simplest which still captures the essential features of heterogeneous capital goods. There is a single final good, $Y(t)$, each unit of which can be allocated to provide either a unit of consumption or a unit of capital of type $k$. Each unit of capital of type $k$ has the following properties: (1) to be fully utilized it requires labor input at the rate $1 / k$, and (2) when fully utilized it produces final output at the rate $f(k) / k$. In other words $k$ is simply the capital-labor ratio (where capital is measured in terms of original or replacement cost), and $f(k)$ the output-labor ratio associated with a unit of capital of type $k$. The totality of techniques available is therefore completely specified by the function $f(k)$ for $k \geqq 0$. For convenience, as well as ease of comparison with the malleable capital model, we assume that $f$ is a standard neoclassical production function; that is, we assume that $f$ is increasing, strictly concave, twice continuously differentiable, and satisfies the endpoint conditions $f(0)=0, f^{\prime}(0)=\infty, f(\infty) \leqq \infty$, and $f^{\prime}(\infty)=0$.

As the techniques available are unchanging over time, at any point in time all relevant information about the past behavior of the economy is summarized in the available amount of, or, what amounts to the same thing, possible employment on capital of each type. In order to allow for uneveness in the choices of technique for past investment, it is useful to 
denote the latter quantities in terms of a distribution rather than a densitymass function. Thus, we denote by $N(k, t)$ possible employment on all types of capital less than $k$ at time $t$. In general, $N$ may have unequal left and right-hand derivatives (for adjacent types of capital in which there has been uneven past investment), flat segments (for an interval of types of capital in which there has been no past investment), and even discontinuities (for a type of capital in which there has been extensive past investment). For the most part, however, we will carry out the analysis under the assumption that there are neither flat segments nor discontinuities (in the relevant domain of $k$ ), merely indicating how the analysis would have to be modified otherwise. In particular, for the behavioral hypotheses we will consider, this assumption would be satisfied for $t \geqq 0$ if it were satisfied for $t=0$.

Now, given possible employment at any point in time, $N(k, t)$, efficient employment of the labor force at that point, $N(t)$, requires that if a type machine $k^{\prime}$ is used then all machines of the type $k>k^{\prime}$ be used; otherwise the rate of final output could be increased by shifting labor from the machine of type $k^{\prime}$ to some machine of type $k>k^{\prime}$. This just means that efficient short-run allocation in this economy is fully described by the condition

$$
N(t)=\int_{\mathbf{k}(t)}^{\infty} d N(k, t)=N(\infty, t)-N[\mathbf{k}(t), t],^{1}
$$

defining the marginal type of machine utilized $\mathbf{k}(t)$. But this condition is also just the description of equilibrium in a competitive labor market in which the wage rate $w(t)$ is bid to settle at the output-labor ratio of the marginal type machine utilized

$$
w(t)=f[k(t)] .^{2}
$$

${ }^{1}$ Clearly we assume that full employment rather than capacity utilization is the operative constraint in this economy. If full employment occurs at the upper endpoint of a flat segment, say $\left(k^{\prime}, \mathbf{k}(t)\right)$, or at a discontinuity, then the condition (2.1) becomes

or

$$
N(t)=\int_{k \in\left(k^{\prime}, \mathbf{k}(t)\right)}^{\infty} d N(k, t)
$$

$$
\lim _{x \rightarrow \mathbf{k}(t)-} \int_{x}^{\infty} d N(k, t) \leqq N(t) \leqq \lim _{x \rightarrow \mathbf{k}(t)+} \int_{x}^{\infty} d N(k, t) .
$$

${ }^{2}$ The wage rate will be indeterminate when full employment occurs at the upper endpoint of a flat segment. Possible market mechanisms which avoid this indeterminacy may or may not be tractable to analysis, as the sketchy discussion following Theorem 3.3 indicates. 
Moreover, it immediately yields the rate of final output $Y(t)$ as the aggregate of rates from all types of machine utilized;

$$
Y(t)=\int_{\mathbf{k}(t)}^{\infty} f(k) d N(k, t)=[f(k) N(k, t)]_{\mathbf{k}(t)}^{\infty}-\int_{\mathbf{k}(t)}^{\infty} f^{\prime}(k) N(k, t) d k .
$$

To complete the model three more elements are required: (1) a labor supply function, (2) a description of aggregate saving behavior, and (3) a characterization of the choice of technique.

For the first we adopt the conventional assumption that the labor force is supplied inelastically and grows at a constant exogenous rate,

$$
\dot{N}(t)=n N(t) \text { with } n \geqq 0 .^{3}
$$

As usual, this assumption implies that, if all the real variables in this economy are now measured in terms of the labor supply, then the only substantive effect for the analysis is that all growth rates are reduced by $n$. To gain this simplification we henceforth redefine all real variables, including the labor supply itself, to be so measured.

To describe aggregate saving behavior, in the bulk of the paper we adopt the hypothesis that gross saving is the sum of a constant fraction saved from profits plus a constant fraction saved from wages:

$$
I(t)=s_{\pi}[Y(t)-w(t)]+s_{w} w(t)=s_{\pi} Y(t)+\left(s_{w}-s_{\pi}\right) w(t)
$$

with

$$
0 \leqq s_{\pi}, s_{w} \leqq 1,
$$

with special attention devoted to the particular case where these saving rates are equal, $0<s=s_{\pi}=s_{w}<1$. However, in Part V we shall explore the implications of the alternative hypothesis that gross saving is determined, say, by a central planning authority, to maximize the discounted sum of all future consumption per capita:

$$
\operatorname{maximize} \int_{0}^{\infty} C(t) e^{-\delta t} d t \text { with } \delta>0 .
$$

Finally, regarding the choice of technique we shall consider three hypotheses, each assuming that investing units choose the capital-labor ratio associated with current investment $\hat{k}(t)$ to maximize the present value of their return, but differing in the manner by which investing units formulate their expectations about future prices, wages, and interest rates. These are, in order of their appearance in the sequel, the hypothesis based on the most naïve formulation of expectations-static expectations; the

\footnotetext{
${ }^{3}$ Clearly it makes no difference to the analysis if we conceive of the labor supply as being measured in efficiency units and accordingly interpret its growth rate as being composed of contributions from both expansion of the labor force and improvement in labor efficiency.
} 
hypothesis based on the most sophisticated formulation of expectationsperfect foresight; and an intermediate hypothesis based on a probabilistic formulation of expectations. The details of these hypotheses will be discussed later.

Given some aggregate saving behavior, $I(t)$, and some choice of technique, $\hat{k}(t)$, it is easily seen that the evolution of the employment distribution function $N(k, t)$ is given by the partial differential equations:

$$
\begin{aligned}
\frac{\partial N(k, t)}{\partial t} & =-\lambda N(k, t), \quad k \leqq \hat{k}(t) ; \\
& =-\lambda N(k, t)+\frac{I(t)}{\hat{k}(t)}, k>\hat{k}(t) ;
\end{aligned}
$$

where, if we introduce a constant exogenous rate of capital depreciation $\mu \geqq 0$, then $\lambda=n+\mu$ represents the rate of loss of relative employment on a machine of any type due to growth of the labor supply and depreciation of the capital stock.

The foregoing system, along with a specification of the initial employment distribution function, provides a complete description of the behavior of the economy over time. Our qualitative analysis of this behavior will rely heavily on the following two properties of this system.

$$
\text { Property } 1: \quad \dot{w}(t) \gtreqless 0 \quad \text { according as } \frac{I(t)}{\hat{k}(t)}-\lambda \gtreqless 0 .^{4}
$$

Whether the wage rate is increasing or decreasing depends only on whether more or less employment is being supplied by new investment than is being demanded by labor force growth and capital stock depreciation.

$$
\text { Property 2: } \quad \dot{Y}(t)=f[\hat{k}(t)] \frac{I(t)}{\hat{k}(t)}-\lambda Y(t)-w(t)\left[\frac{I(t)}{\hat{k}(t)}-\lambda\right] .
$$

This is a similarly interpreted statement about the change in output. ${ }^{5}$ When $N(k, t)$ is differentiable at $k=\mathbf{k}(t)$, these properties can be derived by straightforward differentiation of (2.1) and (2.3), respectively. Thus,

${ }^{4} \dot{w}(t)$ should be interpreted as

$$
\lim _{\Delta t \rightarrow 0^{+}} \frac{w(t+\Delta t)-w(t)}{\Delta t}
$$

When $N(k, t)$ is not differentiable from the right at $k=k(t)$, Property 1 should read $w(t+\Delta t)-w(t) \gtrless 0$ according as $[I(t) / \hat{k}(t)]-\lambda \gtrless 0$ for some $\epsilon>0$ and all $0<\Delta t$ $<\epsilon$.

${ }^{5}$ The statement of both properties assumes $\mathbf{k}(t) \leqq \hat{k}(t)$, that is, the new investment is utilized immediately. If this were not the case, then clearly $\dot{w}<0$ and $\dot{y}=$ $-\lambda(y-w)$. 
recalling the convention adopted earlier that all variables are measured in per capita terms, we obtain upon differentiating (2.1):

$$
\begin{aligned}
0 & =\frac{\partial N(\infty, t)}{\partial t}-\frac{\partial N[\mathbf{k}(t), t]}{\partial t}-\frac{\partial N[\mathbf{k}(t), t]}{\partial \mathbf{k}(t)} \dot{\mathbf{k}}(t) \\
& =\frac{I(t)}{\hat{k}(t)}-\lambda-\frac{\partial N[\mathbf{k}(t), t]}{\partial \mathbf{k}(t)} \frac{\dot{w}(t)}{f^{\prime}[\mathbf{k}(t)]}
\end{aligned}
$$

or

$$
\dot{w}(t)=\frac{\{[I(t) \mid \hat{k}(t)]-\lambda\} \mid f^{\prime}[k(t)]}{\frac{\partial N[\mathbf{k}(t), t]}{\partial \mathbf{k}(t)}},
$$

from which Property 1 follows immediately. Similarly, differentiation of (2.3) (and substitution for $\{\partial N[\mathbf{k}(t), t] / \partial \mathbf{k}(t)\} \mathbf{k}(t)$ from above) yields Property 2:

$$
\begin{aligned}
\dot{Y}(t)= & -\left\{f[\mathbf{k}(t)] \frac{\partial N[\mathbf{k}(t), t]}{\partial \mathbf{k}(t)}+f^{\prime}[\mathbf{k}(t)] N[\mathbf{k}(t), t]\right\} \mathbf{k}(t)+\left[f(k) \frac{\partial N(k, t)}{\partial t}\right]_{\mathbf{k}(t)}^{\infty} \\
& +f^{\prime}[\mathbf{k}(t)] N[\mathbf{k}(t), t] \dot{\mathbf{k}}(t)-\int_{\mathbf{k}(t)}^{\infty} f^{\prime}(k) \frac{\partial N(k, t)}{\partial t} d k \\
= & f[\hat{k}(t)] \frac{I(t)}{\hat{k}(t)}-\lambda Y(t)-w(t)\left[\frac{I(t)}{\hat{k}(t)}-\lambda\right] .
\end{aligned}
$$

When $N(k, t)$ is not differentiable at $k=\mathbf{k}(t)$, the story is slightly more complicated. Consider first the derivation of (2.7). This follows directly from an analysis of the difference quotient for $N(t)$ defined from equation (2.1) (again recalling that we have assumed $N[t]$ identically equal to one):

$$
\begin{aligned}
& 0= \frac{\int_{\mathbf{k}(t+\Delta t)}^{\infty} d N(k, t+\Delta t)-\int_{\mathbf{k}(t)}^{\infty} d N(k, t)}{\Delta t} \\
&= \frac{\{N(\infty, t+\Delta t)-N[\mathbf{k}(t+\Delta t), t+\Delta t]\}}{-\{N(\infty, t)-N[\mathbf{k}(t), t]\} \pm N[\mathbf{k}(t), t+\Delta t]} \\
&=\left\{\frac{N(\infty, t+\Delta t)-N(\infty, t)}{\Delta t}-\frac{N[\mathbf{k}(t), t+\Delta t]-N[\mathbf{k}(t), t]}{\Delta t}\right\} \\
&-\frac{N[\mathbf{k}(t+\Delta t), t+\Delta t]-N[\mathbf{k}(t), t+\Delta t]}{\Delta t}
\end{aligned}
$$

Assuming that $\mathbf{k}(t) \leqq \hat{k}(t)$, from (2.6) the limit of the first and hence the second term in the last expression is just $[I(t) \mid \hat{k}(t)]-\lambda$. But, because by 
assumption, $N(k, t)$ is strictly increasing near $\mathbf{k}(t)$ (that is, $N[k, t]$ has no relevant flat segments), this entails the asserted property.

Property 2 also follows from a consideration of the appropriate difference quotient, now for $Y(t)$ defined from equation (2.3).

$$
\begin{aligned}
& \frac{Y(t+\Delta t)-Y(t)}{\Delta t} \\
& =\frac{\int_{\mathbf{k}(t+\Delta t)}^{\infty} f(k) d N(k, t+\Delta t)-\int_{\mathbf{k}(t)}^{\infty} f(k) d N(k, t)}{\Delta t} \\
& =\frac{\int_{\mathbf{k}(t)}^{\infty} f(k)[d N(k, t+\Delta t)-d N(k, t)]+\int_{\mathbf{k}(t+\Delta t)}^{\mathbf{k}(t)} f(k) d N(k, t+\Delta t)}{\Delta t} \\
& =\int_{\mathbf{k}(t)}^{\infty} f(k) \frac{d N(k, t+\Delta t)-d N(k, t)}{\Delta t}+f(\tilde{k}) \frac{\int_{\mathbf{k}(t+\Delta t)}^{\mathbf{k}(t)} d N(k, t+\Delta t)}{\Delta t} \\
& =\left\{\left[f(k) \frac{N(k, t+\Delta t)-N(k, t)}{\Delta t}\right]_{\mathbf{k}(t)}^{\infty}-\int_{\mathbf{k}(t)}^{\infty} f^{\prime}(k) \frac{N(k, t+\Delta t)-N(k, t)}{\Delta t} d k\right\} \\
& -f(\tilde{k}) \frac{N[\mathbf{k}(t+\Delta t), t+\Delta t]-N[\mathbf{k}(t), t+\Delta t]}{\Delta t} \quad(2.10)
\end{aligned}
$$

for some $\tilde{k}$ between $\mathbf{k}(t+\Delta t)$ and $\mathbf{k}(t)$ by the mean value theorem for integrals. But from (2.6) the limit of the first term in the last expression can be shown, after some analysis, to be simply $f[\hat{k}(t)][I(t) / \hat{k}(t)]-\lambda Y(t)$, while from (2.8) the limit of the second term in the last expression is $f[\mathbf{k}(t)]$ $\times\{[I(t) \mid \hat{k}(t)]-\lambda\}$. Thus, Property 2 is also verified.

We should mention that these properties must be modified slightly when flat segments or discontinuities in $N(k, t)$ are permitted. ${ }^{6}$ The really crucial point about the present model, however, is that regardless of the complexity of past history or present behavior, these properties will enable us to an-

${ }^{6}$ When full employment occurs at the upper endpoint of a flat segment, the requisite modification depends on the particular market mechanism for avoiding indeterminancy in the wage rate which is postulated. When full employment occurs at a discontinuity, these properties must be modified to let $\dot{w}(t)=0$ and $\dot{Y}(t)=f[\hat{k}(t)]$ $\times[I(t) / \hat{k}(t)]-\lambda Y(t)$ whenever

ii)

$$
\lim _{x \rightarrow \mathbf{k}^{(}(t)-} \int_{x}^{\infty} d N(k, t)<N(t)<\lim _{x \rightarrow \mathbf{k}(t)} \int_{x}^{\infty} d N(k, t),
$$

or iii)

$$
\lim _{x \rightarrow \mathbf{k}(t)-} \int_{x}^{\infty} d N(k, t)=N(t) \text { and } \frac{I(t)}{\hat{k}(t)}-\lambda>0
$$

$$
N(t)=\lim _{x \rightarrow \mathbf{k}(t)+} \int_{x}^{\infty} d N(k, t) \text { and } \frac{I(t)}{\hat{k}(t)}-\lambda<0 .
$$


alyze the qualitative behavior of the economy as if it were completely described by a system of ordinary differential equations (in contrast to the rather complicated mixed system of equations by which the economy is actually described).

\section{Static Expectations}

In this section we investigate the behavior of the economy when aggregate saving behavior is described by (2.5a) and investing units expect future wage and interest rates (with final output as the numeraire good) to be the same as those prevailing today.

Consider a representative investing unit which has at the present time $t$ one unit of final output to invest. For any machine of type $k$ with $f(\mathbf{k}) \geqq$ $w(t)$ this unit expects to earn quasi-rents $\{[f(k)-w(t)] / k\} e^{-\mu(v-t)}$ at any time $v \geqq t$, while, say, on a government bond costing one unit of final output it expects to earn interest $r(t)$ at any time $v \geqq t$. (The latter is best thought of as a convenient heuristic device for introducing the concept of the rate of interest in this context). Hence it can calculate what it believes to be the present value of any machine of type $k$ with $f(k) \geqq w(t)$ :

$$
\int_{t}^{\infty} \frac{f(k)-w(t)}{k} e^{-[r(t)+\mu](v-t)} d v=\frac{f(k)-w(t) /[r(t)+\mu]}{k} .
$$

Assuming the unit chooses $\hat{k}(t)$, the machine of that type which maximizes this present value, its choice is simply described by the machine whose ex ante marginal productivity of labor $g^{\prime}(k)$ is equal to the current wage rate

$$
g^{\prime}[\hat{k}(t)] \equiv f[\hat{k}(t)]-f^{\prime}[\hat{k}(t)] \hat{k}(t)=w(t),
$$

as by the concavity of $f$ :

$$
\frac{d\left[\frac{f(k)-w(t)}{k}\right]}{d k}=\frac{w(t)-g^{\prime}(k)}{k^{2}} \gtreqless 0, \text { according as } k \leqq \hat{k}(t) .
$$

This means, among other things, that the interest rate which makes the investing unit indifferent between capital and bonds, or the present value of a machine of type $\hat{k}(t)$ equal to its cost, must be the net quasi-rent on a machine of type $\hat{k}(t), r(t)=[\{f[\hat{k}(t)]-w(t)\} \mid \hat{k}(t)]-\mu$.

Given the aggregate saving behavior $(2.5 a)$ and the choice of technique (3.2), the qualitative behavior of the economy, summarized by the qualitative behavior of $Y(t)$ and $w(t)$, depends basically on whether $s_{\pi} \geqq s_{w}$ (the "normal" case) or $s_{\pi}<s_{w}$. This can be easily seen from the typical phase diagrams for the two cases (Fig. 1), or, better yet, from the extreme phase diagrams for the two cases (Fig. 2). In brief, we can derive the following conclusions: (1) if $s_{\pi}>s_{w}$, then the economy converges asymptotically to 


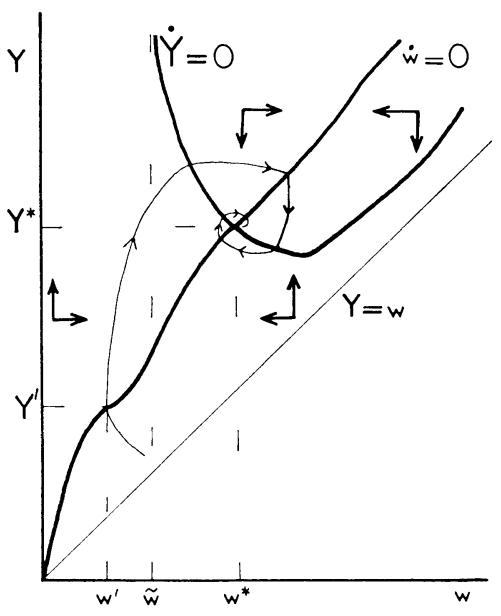

a. $0<s_{w}<s_{\Pi} \leqq 1$

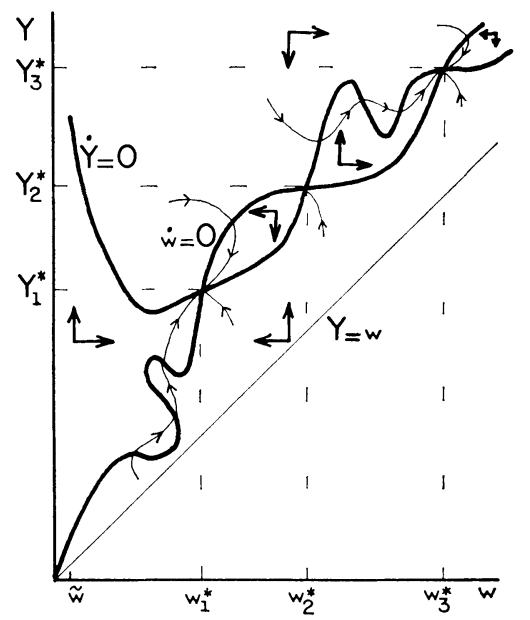

b. $0<s_{\pi}<s_{w} \leqq 1$

FIG. 1

a unique balanced growth path, possibly through continued reinvestment cycles; (2) if $s_{\pi}=s_{w}$, then the economy also converges to a unique balanced growth path, but through at most one-half of a single reinvestment cycle; and (3) if $s_{\pi}<s_{w}$, then the economy converges to possibly one of several balanced growth paths, again through at most one-half of a single reinvestment cycle. ${ }^{7}$

These conclusions follow directly from an analysis of the properties

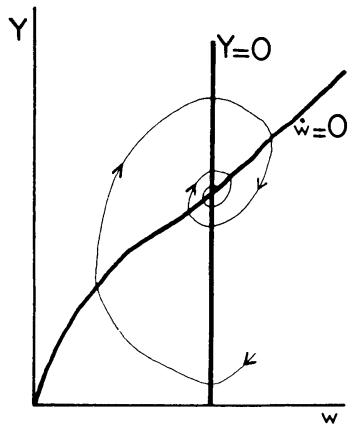

a $0=S_{W}<S_{\pi} \leqq 1$

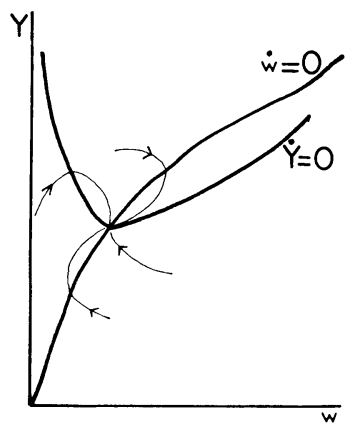

b. $0<S_{n}=S_{w}<1$

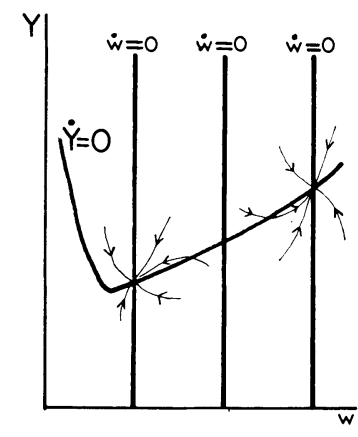

c. $0=S_{\pi}<S_{w} \leqq 1$

FIG. 2

7 "Reinvestment cycle" is a shorthand reference to oscillation in both $Y$ and $w$. The choice of terminology is dictated by the fact that in this model, the possibility of continued oscillation is associated with a larger share of profits than wages being saved $\left(s_{\pi}>s_{w}\right)$. Notice from Fig. $1 b$ that in the opposite case $\left(s_{\pi}<s_{w}\right)$ either $Y(t)$ or $w(t)$, but not both together, may oscillate over some limited period. 
described in Part II, (2.7) and (2.8), under the hypotheses (2.5a) and (3.2), ${ }^{8}$ which we rewrite for this purpose as (hereafter suppressing the time variable $t$ when no confusion may result from doing so):

$$
\dot{w} \gtreqless 0, \text { according as } \frac{I}{\hat{k}}-\lambda=\frac{s_{\pi} Y+\left(s_{w}-s_{\pi}\right) w}{\hat{k}}-\lambda \gtreqless 0,
$$

and

$$
\dot{Y}=f(\hat{k}) \frac{I}{\hat{k}}-\lambda Y-w\left(\frac{I}{\hat{k}}-\lambda\right)=\left[s_{\pi} f^{\prime}(\hat{k})-\lambda\right](Y-w)+s_{w} f^{\prime}(\hat{k}) w,
$$

where

$$
\hat{k}=g^{\prime-1}(w), \quad \text { with } \frac{d \hat{k}}{d w}=\frac{1}{g^{\prime \prime}(\hat{k})}=\frac{1}{-f^{\prime \prime}(\hat{k}) \hat{k}}>0 .
$$

Consider first (3.3). By utilizing the properties of $f$ and the relation (3.5), we find that

$\lim _{w \rightarrow 0}(Y)_{\dot{w}=0}=0$,

$$
\begin{aligned}
(Y)_{\dot{w}=0} & =\frac{\left(s_{\pi}-s_{w}\right) w+\lambda \hat{k}}{s_{\pi}} \\
& =f(\hat{k})-\frac{s_{\pi} f^{\prime}(\hat{k}) \hat{k}+s_{w} w-\lambda \hat{k}}{s_{\pi}} ;
\end{aligned}
$$

$$
\begin{gathered}
\lim _{w \rightarrow \infty}(Y)_{\dot{w}=0}=\lim _{\hat{k} \rightarrow \infty}\left\{\left(\frac{s_{\pi}-s_{w}}{s_{\pi}}\right)\left[\frac{f(\hat{k})}{\hat{k}}-f^{\prime}(\hat{k})\right]+\frac{\lambda}{s_{\pi}}\right\} \hat{k}=\infty ; \\
\left(\frac{d Y}{d w}\right)_{\dot{w}=0}=\frac{s_{\pi}-s_{w}}{s_{\pi}}+\frac{\lambda}{s_{\pi}} \frac{d \hat{k}}{d w}
\end{gathered}
$$

and

if $\dot{w}=0$ at $\left(Y^{\prime}, w^{\prime}\right)$, then $\dot{w} \gtrless 0$ at $\left(\mathrm{Y}^{\prime \prime}, w^{\prime}\right)$, according as $Y^{\prime \prime} \gtrless Y^{\prime}$.

Similarly, from (3.4) we find that

if $\dot{Y}=0($ and $Y>w)$, then $\hat{k}>\tilde{k}$ (or $w>\tilde{w})$ where $s_{\pi} f^{\prime}(\tilde{k})=\lambda$

$$
\begin{aligned}
& {\left[\text { or } \tilde{w}=g^{\prime}(\tilde{k})\right] ; } \\
&(Y)_{\dot{Y}=0}=\left[1-\frac{s_{w} f^{\prime}(\hat{k})}{s_{\pi} f^{\prime}(\hat{k})-\lambda}\right] w ; \\
& \lim _{w \rightarrow \tilde{w}^{+}}(Y)_{\dot{Y}=0}=\infty, \lim _{w \rightarrow \infty}(Y)_{\dot{Y}=0}=\infty ;
\end{aligned}
$$

${ }^{8}$ Our analysis assumes $0<s_{\pi}, s_{w}$. The limiting cases $s_{w}=0$ and $s_{\pi}=0$, as can be seen from Figs. $2 a$ and $2 c$, are essentially extreme examples of the general cases $s_{\pi}>s_{w}$ and $s_{\pi}<s_{w}$, respectively. 


$$
\begin{aligned}
\left(\frac{d Y}{d w}\right)_{\dot{Y}=0} & =\left[1-\frac{s_{w} f^{\prime}(\hat{k})}{s_{\pi} f^{\prime}(\hat{k})-\lambda}\right]+\frac{\lambda s_{w} w f^{\prime \prime}(\hat{k})}{\left[s_{\pi} f^{\prime}(\hat{k})-\lambda\right]^{2}} \frac{d \hat{k}}{d w} \\
& =\frac{Y}{w}+\left[\frac{\lambda}{s_{\pi} f^{\prime}(\hat{k})-\lambda}\right]\left[\frac{Y-w}{f^{\prime}(\hat{k}) \hat{k}}\right] ;
\end{aligned}
$$

and

if $\dot{Y}=0$ at $\left(Y^{\prime}, w^{\prime}\right)$, then $\dot{Y} \lesseqgtr 0$ at $\left(Y^{\prime \prime}, w^{\prime}\right)$, according as $Y^{\prime \prime} \gtrless Y^{\prime}$.

Finally, using (3.3) in evaluating (3.4) yields the result that

$\dot{w}=0$ and $\dot{Y}=0$ if and only if

$$
(Y)_{\dot{w}=0}=(Y)_{\dot{Y}=0}=f(\hat{k}) \text { for } \hat{k}>\tilde{k}(\text { or } w>\tilde{w}) .
$$

Given these preliminary results, we come to the core of the argument, which can be succinctly expressed in three propositions:

\section{Theorem 3.1}

If $\mathrm{s}_{\pi} \geqq \mathrm{s}_{\mathrm{w}}$, then there exists a unique balanced growth path $\left(\mathrm{Y}^{*}, \mathrm{w}^{*}, \hat{\mathrm{k}}^{*}\right)$; if $\mathrm{s}_{\pi}<\mathrm{s}_{\mathrm{w}}$, then there exists at least one balanced growth path and possibly several balanced growth paths $\left(\mathrm{Y}_{1}^{*}, \mathrm{w}_{1}^{*}, \hat{\mathrm{k}}_{1}^{*}\right) \ldots\left(\mathrm{Y}_{\mathrm{m}}^{*}, \mathrm{w}_{\mathrm{m}}^{*}, \hat{\mathrm{k}}_{\mathrm{m}}^{*}\right)$ where $\mathrm{m} \geqq 1$.

\section{Proof}

By virtue of the second right-hand expression in (3.6) and the result (3.15), we need only show that the function

$$
h(w)=s_{\pi} f^{\prime}(\hat{k}) \hat{k}+s_{w} w-\lambda \hat{k}
$$

has a single zero for $w \epsilon(\tilde{w}, \infty)$ when $s_{\pi} \geqq s_{w}$, or at least a single zero and possibly several zeros for $w \epsilon(\tilde{w}, \infty)$, when $s_{\pi}<s_{w}$. But this conclusion follows immediately from the facts that

$\lim _{w \rightarrow \tilde{w}} h(w)=s_{w} \tilde{w}$,

$$
\lim _{w \rightarrow \infty} h(w)=\lim _{\hat{k} \rightarrow \infty}\left\{s_{w}\left[\frac{f(\hat{k})}{\hat{k}}-f^{\prime}(\hat{k})\right]+\left[s_{\pi} f^{\prime}(\hat{k})-\lambda\right]\right\} \hat{k}=-\infty ;
$$

and

$h^{\prime}(w)=\left[s_{\pi} f^{\prime}(\hat{k})-\lambda+s_{\pi} f^{\prime \prime}(\hat{k}) \hat{k}\right] \frac{d \hat{k}}{d w}+s_{w}=\left[s_{\pi} f^{\prime}(\hat{k})-\lambda\right] \frac{d \hat{k}}{d w}+\left(s_{w}-s_{\pi}\right)$, as the last is unambiguously negative for $w \in[\tilde{w}, \infty)$ only if $s_{\pi} \geqq s_{w}$.

\section{Theorem 3.2}

If $\mathrm{s}_{\pi}>\mathrm{s}_{\mathrm{w}}\left(\mathrm{s}_{\pi}=\mathrm{s}_{\mathrm{w}}\right)$, then there is $a \mathrm{w}^{\prime}>\mathrm{w}^{*}\left(\mathrm{w}^{\prime}=\mathrm{w}^{*}\right)$ such that $(\mathrm{dY} / \mathrm{dw})_{\dot{\mathrm{Y}}=0}$ $\lesseqgtr 0$, according as $\mathrm{w} \lesseqgtr \mathrm{w}^{\prime}$; if $\mathrm{s}_{\pi}<\mathrm{s}_{\mathrm{w}}$ then $(\mathrm{dY} / \mathrm{dw})_{\dot{\mathrm{Y}}=0}>0$ whenever $(\mathrm{Y})_{\dot{\mathrm{Y}}=0} \leqq \mathrm{f}(\hat{\mathrm{k}})$ or $(\mathrm{Y})_{\dot{\mathrm{w}}=0} \geqq \mathrm{f}(\hat{\mathrm{k}})$. 
This proposition describes the circumstances under which there can be oscillation in $Y$ : The first part simply means that $Y$ oscillates if and only if $w$ oscillates when $s_{\pi}>s_{w}$ ( $Y$ cannot oscillate when $s_{\pi}=s_{w}$ ), as by (3.8) $(d Y \mid d w)_{\dot{w}=0}>0$ for $s_{\pi} \geqq s_{w}$. The second part is somewhat more complicated; its essence, however, is that if $Y$ oscillates, then $w$ must be increasing when $s_{\pi}<s_{w}$, as by (3.5) $(d Y / d w)_{Y=f(\hat{k})}>0$. This result rules out continued oscillation in $Y$ when $s_{\pi}<s_{w}$. Both parts are illustrated in the exaggerated blowups of portions of Fig. 1 in Fig. 3 below.

\section{Proof}

Let $\tilde{k}>\tilde{k}($ or $\tilde{w}>\tilde{w})$ be defined by $s_{\pi} f(\tilde{\tilde{k}})=\lambda \tilde{k}\left(\right.$ or $\left.\tilde{w}=g^{\prime}(\tilde{k})\right)$. Then, from the second right-hand expression in (3.6) and the result (3.15) we have,

$$
\text { if } s_{\pi}=s_{w} \text {, then }\left(Y^{*}, w^{*}, \hat{k}^{*}\right)=(\tilde{\tilde{Y}}, \tilde{w}, \tilde{\tilde{k}}) \text {. }
$$

Moreover, from (3.11) we have

$$
\frac{\partial\left[(Y)_{\dot{Y}=0}\right]}{\partial s_{w}}=-\frac{w f^{\prime}(\hat{k})}{s_{\pi} f^{\prime}(\hat{k})-\lambda}>0,
$$

which together with

$$
\left[\frac{d Y}{d w}\right]_{Y=f(\hat{\kappa})}=f^{\prime}(\hat{k}) \frac{d \hat{k}}{d w}>0,
$$

implies if $s_{\pi}>s_{w}$, then $\left(Y^{*}, w^{*}, \hat{k}^{*}\right)<(\tilde{Y}$, $\tilde{w}, \tilde{k})$, or if $s_{\pi}<s_{w}$, then $\left(Y_{i}^{*}, w_{i}^{*}, \hat{k}_{i}^{*}\right)>(\tilde{Y}, \tilde{w}, \hat{k})$ for $i=1, \ldots, m$. The first part of the proposition now follows directly from the results, the details of whose derivation we omit, if $s_{\pi}>s_{w}\left(s_{\pi}=s_{w}\right)$, then

$$
\begin{array}{rlrl}
\left(\frac{d Y}{d w}\right)_{\dot{Y}=0} & <0(=0) & \text { for } & w=w^{*}, \\
& >0 & \text { for } \quad w>\tilde{\tilde{w}}
\end{array}
$$

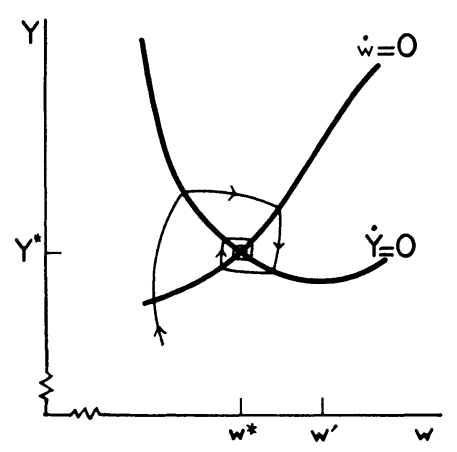

a. $s_{\pi}>s_{w}$

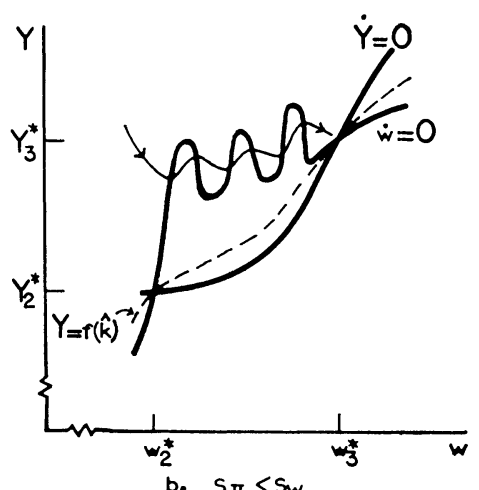

b. $s_{\pi}<s_{W}$

FIG. 3 
and

$$
\left(\frac{d^{2} Y}{d w^{2}}\right)_{\dot{Y}=0}>0 \quad \text { for } \quad w \leqq \tilde{w} .
$$

Similarly, the second part follows from the results, whose details we also omit, if $s_{\pi}<s_{w}$, then $(Y)_{\dot{w}=0}<f(\hat{k})$ for $w<w_{i}^{*}$, and $(D Y / d w)_{\dot{Y}=0}>0$ for $w \geqq \tilde{w}$ and either $(Y)_{\dot{Y}=0} \leqq f(\hat{k})$ or $(Y)_{\dot{w}=0} \geqq f(\hat{k})$.

\section{Theorem 3.3}

If $\mathrm{s}_{\pi}>\mathrm{s}_{\mathrm{w}}$, then

$$
\lim _{\mathrm{t} \rightarrow \infty}[\mathrm{Y}(\mathrm{t}), \mathrm{w}(\mathrm{t}), \hat{\mathrm{k}}(\mathrm{t})]=\left(\mathrm{Y}^{*}, \mathrm{w}^{*}, \hat{\mathrm{k}}^{*}\right) .
$$

It should be clear that convergence to a balanced growth path when $s_{\pi}$ $\leqq s_{w}$ is assured by the fact that continued oscillation in $Y$ or $w$ is impossible.

\section{Proof}

We must show that periodic or undamped oscillation around the balanced growth path is impossible. The proof involves a contradiction. Suppose at time $t^{\prime} \geqq 0$ we observe $\dot{Y}\left(t^{\prime}\right)>0$ and $\dot{w}\left(t^{\prime}\right)=0$ at $\left[Y\left(t^{\prime}\right), w\left(t^{\prime}\right)\right]=\left(Y^{\prime}, w^{\prime}\right)$ $<\left(Y^{*}, w^{*}\right)$ (see Fig. 1a above). Now periodic or undamped oscillation would require both this and that at some later time $t^{\prime \prime}>t^{\prime} Y\left(t^{\prime \prime}\right) \leqq Y^{\prime}$ and $w\left(t^{\prime \prime}\right)=w^{\prime}$ with $w(t)>w^{\prime}$ for $t^{\prime}<t<t^{\prime \prime}$. Suppose then that the latter is also the case. Then static expectations (3.5) imply that $k(t) \geqq k^{\prime}$ for $t^{\prime} \leqq t \leqq t^{\prime \prime}$. In addition the properties (3.3) and (3.4) imply that

$$
\dot{Y}\left(t^{\prime}\right)=f\left[\hat{k}\left(t^{\prime}\right)\right] \frac{I\left(t^{\prime}\right)}{\hat{k}\left(t^{\prime}\right)}-\lambda Y\left(t^{\prime}\right)>\dot{w}\left(t^{\prime}\right)=\frac{I\left(t^{\prime}\right)}{\hat{k}\left(t^{\prime}\right)}-\lambda=0,
$$

or $f\left(\hat{k}^{\prime}\right)>Y^{\prime}$. Utilizing these facts along with the underlying technological relationships (2.1), (2.3), and (2.6), we see that the following must be true:

$$
\begin{aligned}
Y\left(t^{\prime \prime}\right) & =\int_{\mathbf{k}^{\prime}}^{\infty} f(k) d N\left(k, t^{\prime \prime}\right) \\
& =\int_{\mathbf{k}^{\prime}}^{\infty} f(k)\left[d N\left(k, t^{\prime \prime}\right)-d N\left(k, t^{\prime}\right) e^{-\lambda\left(t^{\prime \prime}-t^{\prime}\right)}\right]+\int_{\mathbf{k}^{\prime}}^{\infty} f(k) d N\left(k, t^{\prime}\right) e^{-\lambda\left(t^{\prime \prime}-t^{\prime}\right)} \\
& \geqq f\left(\hat{k}^{\prime}\right) \int_{\mathbf{k}^{\prime}}^{\infty}\left[d N\left(k, t^{\prime \prime}\right)-d N\left(k, t^{\prime}\right) e^{-\lambda\left(t^{\prime \prime}-t^{\prime}\right)}\right]+Y^{\prime} e^{-\lambda\left(t^{\prime \prime}-t^{\prime}\right)} \\
& =f\left(\hat{k}^{\prime}\right)\left[1-e^{-\lambda\left(t^{\prime \prime}-t^{\prime}\right)}\right]+Y^{\prime} e^{-\lambda\left(t^{\prime \prime}-t^{\prime}\right)}>Y^{\prime},
\end{aligned}
$$

as, if $\hat{k}(t) \geqq \hat{k}^{\prime}$ for $t^{\prime} \leqq t \leqq t^{\prime \prime}$, then $d N\left(k, t^{\prime \prime}\right)-d N\left(k, t^{\prime}\right) e^{-\lambda\left(t^{\prime \prime}-t^{\prime}\right)}=0$ for $k<\hat{k}^{\prime}$. But this contradicts the original hypothesis that $Y\left(t^{\prime \prime}\right) \leqq Y^{\prime}$. 
A few general comments about the foregoing results are appropriate. First, we note that the behavior of this economy is considerably richer than the behavior of the analogous economy with malleable capital. In particular, though in the long run the two are indistinguishable (as along a balanced growth path, static expectations are in fact fulfilled), in the short run there is very likely to be some degree of oscillation in significant variables here which is impossible there. This difference is clearly quite strongly related to the fact that the profile as well as the aggregate of past behavior impinges on current behavior in this economy and is apparently somewhat weakly related to the particular behavioral hypotheses adopted (as Part IV illustrates).

Second, we mention that stable long-run behavior in this economy depends crucially on the assumption that the exogenous technological influences, capital depreciation and technical change, are exponential. For example, if capital depreciation were abrupt, then replacement cycles would very likely reinforce reinvestment cycles to keep the economy in a perpetual state of oscillation. This observation also suggests that if these factors were made endogenous, then they might also tend to destabilize the behavior of the economy.

Finally, we outline how either flat segments or discontinuities in the initial employment distribution function $N(k, 0)$ affect our results. For example, consider the borderline case $s_{\pi}=s_{w}$, and assume, first, that there is a flat segment for $k \epsilon\left(k^{\prime}, \mathbf{k}(0)\right)$, that is, that there are initially no machines of type $k \epsilon\left(k^{\prime}, \mathbf{k}(0)\right)$, and then, second, there is a discontinuity at $k=\mathbf{k}(0)$, that is, that there is initially a mass of machines of type $k=\mathbf{k}(0)$, as depicted in Figure 4 for $\{Y(0), f(\mathbf{k}(0))\}<\left(Y^{*}, w^{*}\right) .^{9}$

When there is a flat segment for $k \epsilon\left(k^{\prime}, \mathbf{k}(0)\right)$, so that $w(0)$ could fall anywhere between $f\left(k^{\prime}\right)$ and $f(\mathbf{k}(0))$, the natural choice for the initial wage rate is that value which entails an unambiguous determination of its later development; for instance, in Figure $4 a$ we depict the two possibilities for $\{Y(0), f(\mathbf{k}(0))\}<\left(Y^{*}, w^{*}\right)$. On the one hand, there does not seem to be any appealing economic argument to justify this particular choice. On the other, however, it should not be surprising that the natural choice is at the same time an artificial one; this sort of difficulty pervades equilibrium theory. ${ }^{10}$

When there is a discontinuity at $k=\mathbf{k}(0)$, things are much simpler: $w$ just remains constant at $f(\mathbf{k}(0))$ for some period $\left(0, t^{\prime}\right)$. The length of this period $t^{\prime}$ corresponds roughly to the time it takes to build new machines to

${ }^{9}$ The more general situation where the flat segment or discontinuity becomes relevant at some time $t^{\prime}>0$ can be analyzed in a similar fashion once investment during the period $\left(0, t^{\prime}\right)$ is accounted for.

${ }^{10}$ If we follow Marshall's dictum, then the market mechanism appropriate in this dynamic context, gradual rather than abrupt adjustment of the wage rate toward an equilibrating level, avoids an indeterminate wage rate but introduces an unstable one (to say nothing of Keynesian unemployment). 


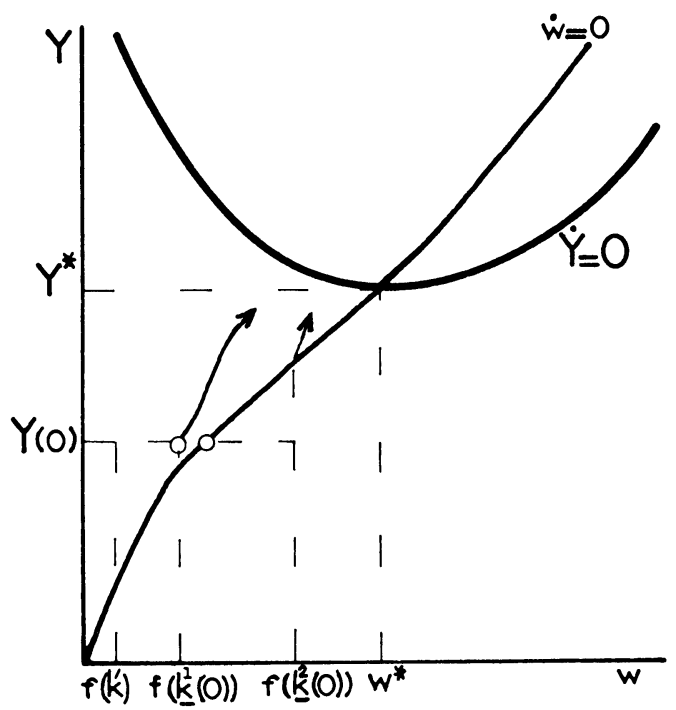

a. $N(k, 0)$ constant for $k \in\left(k^{\prime}, \underline{k}(0)\right)$

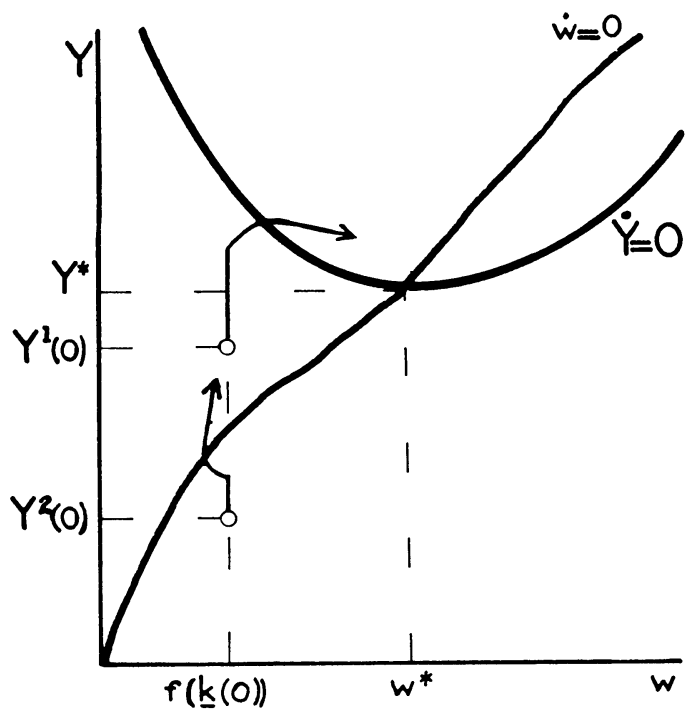

$$
\text { b. } \lim _{k \rightarrow \underline{k}(0)^{-}} N(k, 0)<\lim _{k \rightarrow \underline{k}(0)^{+}} N(k, 0)
$$

FIG. 4 
replace those of type $\mathbf{k}(0)$, or the time it takes for population growth and depreciation to force full utilization of machines of type $\mathbf{k}(0)$. These two situations are depicted by the development of the economy from $\left\{Y^{1}(0)\right.$, $f(\mathbf{k}(0))\}<\left(Y^{*}, w^{*}\right)$ and $\left\{Y^{2}(0), f(\mathbf{k}(0))\right\}<\left(Y^{*}, w^{*}\right)$, respectively, in Figure $4 b$.

\section{Perfect Foresight}

In Part III the investor based his decision on what type of machines to construct on very naïve expectations: he assumed that the wage today would continue indefinitely far into the future. In Part IV we consider the other polar case: the individual is assumed to have perfect foresight, that is, the wage and interest rates he expects today for any given date in the future will in fact be the wage and interest rates at that time. In other words, we consider equilibrium paths, paths along which, if individuals act on their expectations, those expectations are fulfilled.

Let us consider the price system as viewed at time zero. We denote the instantaneous rate of return by $r(t)$, and let

$$
R(t)=\int_{0}^{t} r(\tau) d \tau
$$

Throughout this analysis we shall assume that there is some investment and some consumption going on at every point of time. Thus, competitive equilibrium requires that the price of output must equal the price of consumption which must equal the market price of the type of capital good being constructed at that time. If we let output be the numeraire, then the price of output at time $t$ as viewed at time 0 is simply

$$
p(t)=e^{-R(t)} \text {. }
$$

The price of output at time $v$ as viewed at time $t$ is simply

$$
p(v, t)=p(v) / p(t)=e^{-[R(v)-R(t)]} .
$$

The demand price for a capital good of type $k$ at time $t$ is

$$
q(k, t)=\int_{A(k, t)} \frac{f(k)-w(v)}{k} p(v, t) d v,
$$

where $A$ is defined as the set of intervals over which the machine is used, that is,

$$
A(k, t)=[v \mid v \geqq t ; w(v) \leqq f(k)] .
$$

It is sometimes convenient to write (4.4) in a slightly different form:

$$
q(k, t)=\sum_{i} \int_{v_{i}(k, t)}^{\hat{i}^{(k, t)}} \frac{f(k)-w(v)}{k} p(v, t) d v,
$$


where $\hat{v}_{i}(k, t)$ is defined as the $i$ th value of $v>t$ for which

and

$$
\lim _{v \rightarrow \hat{v}_{i}(k, t)^{-}} w(v) \leqq f(k),
$$

$$
\lim _{v \rightarrow \hat{v}_{t}(k, t)^{+}} w(v) \geqq f(k) .
$$

Similarly, for $k \geqq \mathbf{k}, v_{i}(k, t)$ is defined as the $(i-1)$ th value of $v>t$ for which

and

$$
\lim _{v \rightarrow v_{i}(k, t)^{-}} w(v) \geqq f(k)
$$

$$
\lim _{v \rightarrow v_{i}(k, t)^{+}} w(v) \leqq f(k) \quad \text { for } \quad i \geqq 2,
$$

and as $t$ for $i=1$. (For $k<\mathbf{k}, v_{i}[k, t]$ is defined symmetrically with $\hat{v}_{i}[k, t]$.) Graphically, these definitions can be simply represented as in Figure 5.

The type of machine with the highest demand price is built, since, by assumption, the costs of building all machines are identical. Thus, again denoting this type of machine by $\hat{k}$, it must be that

$$
\frac{\partial q(\hat{k}, t)}{\partial k}=\int_{A(\hat{k}, t)}\left\{\frac{w(v)-\left[f(\hat{k})-\hat{k} f^{\prime}(\hat{k})\right]}{\hat{k}^{2}}\right\} p(v, t) d v=0 ; ;^{11}
$$

or

$$
f(\hat{k})-\hat{k} f^{\prime}(\hat{k})=\frac{\int_{A(\hat{k}, t)} w(v) p(v, t) d v}{\int_{A(\hat{k}, t)} p(v, t) d v} .
$$

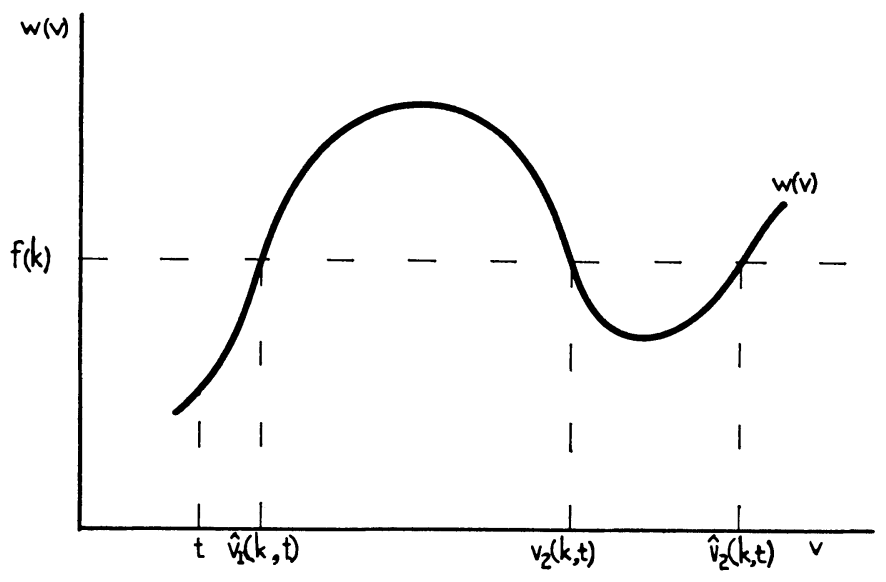

FIG. 5

${ }^{11}$ It is easy to show that this derivative exists and has the indicated value, even if $w(v)$ is not continuous (or differentiable), since either the value of the integrand at the limits of integration in (4.4) is zero or the limits of integration in (4.4) are invariant to small changes in $k$. 
The "weighted average" wage must equal the marginal product of labor.

A sufficient condition for the uniqueness of $\hat{k}$ is the negativity of

$$
\begin{aligned}
\frac{\partial^{2} q(k, t)}{\partial k^{2}}= & -2 \int_{A(k, t)} \frac{w-g^{\prime}(k)}{k^{3}} p(v, t) d v+\int_{A(k, t)} \frac{f^{\prime \prime}(k)}{k} p(v, t) d v \\
& -\frac{\sum_{i}\left\{w\left[v_{i}(k, t)\right]-g^{\prime}(k)\right\} p\left[v_{i}(k, t), t\right]}{k^{2}} \cdot \frac{d v_{i}(k, t)}{\partial k} \\
& +\frac{\sum_{i}\left\{w\left[\hat{v}_{i}(k, t)\right]-g^{\prime}(k)\right\} p\left[\hat{v}_{i}(k, t), t\right]}{k^{2}} \cdot \frac{\partial \hat{v}_{i}(k, t)}{\partial k}
\end{aligned}
$$

where, as before, $g^{\prime}(k)=f(k)-k f^{\prime}(k)$. Note that the first term, by (4.7), is zero if $k=\hat{k}$. The second term is unambiguously negative. But the last two terms are unambiguously positive, since $w\left[v_{i}(k, t)\right]=f(k)$, and $w\left[\hat{v}_{i}(k, t)\right]=f(k) .^{12}$ Hence, we cannot rule out the possibility that there exists more than one $k$ that could be constructed at any given time, that is, such that

$$
q(k, t)=\max _{k \geqq 0}\{q(k, t)\} .
$$

Later on we shall show that, over any interval of time, there can exist at most two such types of machines. For the moment we shall simply assume that only one type of machine is built at any time (and that $\hat{k}[t]$ is continuous).

As we have already indicated, since there is always some consumption and some investment going on, competitive equilibrium requires that

$$
q(\hat{k}, t)=\int_{A(\hat{k}, t)} \frac{f(\hat{k})-w}{\hat{k}} p(v, t) d v=1 .
$$

Subtracting (4.7), we obtain

$$
1=\int_{A(\hat{k}, t)} f^{\prime}(\hat{k}) p(v, t) d v
$$

Notice that (4.10) is identical to the corresponding expression for perfectly malleable capital; the price of capital is equal to the discounted quasi-rents, which in the malleable capital case is just $f^{\prime}(k)$ :

$$
1=\int_{t}^{\infty} f^{\prime}[k(v)] p(v, t) d v
$$

The behavior of the economy is completely described by equations (4.1)-(4.10) and the equations in output and employment, which are

${ }^{12}$ Except if at a given $v_{i}, w(v)$ is not differentiable or continuous. If $w(v)$ is differentiable everywhere except at points of discontinuities, then we sum only over those $i$ 's at which $w\left(v_{i}\right)$ is continuous. If $w(v)$ is not differentiable at some points at which it is continuous, the analysis goes through with the appropriate limit operations. 
identical to those of the previous sections (eq. [2.1] and [2.3]). We shall assume a constant proportion of income, $s$, is saved.

As before, the behavior may be more easily analyzed in the differencedifferential form rather than the integral form.

Differentiating (4.4), we obtain a differential equation for the demand price of a machine of type $k$ :

$$
\frac{\dot{q}(k, t)}{q(k, t)}=r(t)-\frac{f(k)-w(t)}{k q(k, t)} .
$$

By differentiating (4.7) we obtain an equation describing the change in the type of machine constructed $:^{13}$

$$
\frac{\partial^{2} q(\hat{k}, t)}{\partial k^{2}} \dot{\hat{k}}+r(t) \frac{\partial q(\hat{k}, t)}{\partial k}-\frac{w(t)-\left[f(\hat{k})-\hat{k} f^{\prime}(\hat{k})\right]}{\hat{k}^{2}}=0,
$$

or

$$
\dot{\hat{k}}=\frac{w-\left[f(\hat{k})-\hat{k} f^{\prime}(\hat{k})\right]}{\hat{k}^{2} \frac{\partial^{2} q(\hat{k}, t)}{\partial k^{2}}} .
$$

Since, by the second-order condition, the denominator is negative,

$$
\operatorname{sign} \dot{\hat{k}}=\operatorname{sign}\left[f(\hat{k})-\hat{k} f^{\prime}(\hat{k})-w\right]
$$

As before (Part II),

$$
\operatorname{sign} \dot{w}=\operatorname{sign}[-\lambda+(s Y / \hat{k})]
$$

and

$$
\begin{aligned}
\frac{\dot{Y}}{Y} & =-\lambda+\frac{s f(\hat{k})}{\hat{k}}-\frac{w \dot{w} \frac{\partial N(\mathbf{k}, t)}{\partial k}}{Y f^{\prime}(\mathbf{k})} \\
& =-\lambda+\frac{\lambda w}{Y}+s \frac{[f(\hat{k})-w]}{\hat{k}} .
\end{aligned}
$$

\section{Dynamic Behavior}

We now analyze the dynamic behavior of the economy. We will continue the analysis with the assumptions that only one type of machine is constructed at any time and that $\hat{k}(t)$ is continuous. Later, these assumptions will be lifted.

${ }^{13}$ Even if $\left(\partial^{2} q\right) /\left(\partial k^{2}\right)$ does not exist, the sign of $\dot{\hat{K}}$ can be shown to be that of $g^{\prime}(\hat{k})-w$, by arguments similar to those used in Part II. 
We will first show that $Y$ must converge to $Y^{*}$, where, as before, $Y^{*}=$ $f\left(\hat{k}^{*}\right)$ with $s f\left(\hat{k}^{*}\right)=\lambda \hat{k}^{*}$. We will then show that $\hat{k}$ must converge to $\hat{k}^{*}$ and $w$ to $w^{*}$. In establishing that all perfect foresight paths must converge to balanced growth, we will, moreover, be able to determine what the dynamic path looks like.

\section{Lemma 1}

If $\dot{Y}$ is ever non-positive, it is always non-positive. Evaluating $[d(\dot{Y} / Y) / d t]$ at $\dot{Y}=0$, we obtain, ${ }^{14}$

$$
\left[\frac{d(\dot{Y} / Y)}{d t}\right]_{\dot{Y}=0}=\left[\frac{\lambda}{Y}-\frac{s}{\hat{k}}\right] \dot{w}+\frac{s\left[w-g^{\prime}(\hat{k})\right] \dot{\hat{k}}}{\hat{k}^{2}} \leqq 0,
$$

since, as we have already noted, the sign of $\dot{w}$ is identical to that of $-\lambda+$ $(s Y \mid \hat{k})$, while the sign of $\dot{\hat{k}}$ is the same as $g^{\prime}(\hat{k})-w$.

\section{Lemma 2}

If $Y<Y^{*}, \dot{Y}>0$. Since $\dot{Y} \mid Y$ is a function of $w, \hat{k}$, and $Y$, we have $\dot{Y} \mid Y=$ $\psi(w, \hat{k}, Y)$. In Part III, we showed that if $w=g^{\prime}(\hat{k}), \dot{Y}>0$ if $Y<Y^{*}$. If $\dot{Y} \leqq 0$ and $Y<Y^{*}, w$ cannot equal $g^{\prime}(\hat{k})$ and $\psi(w, \hat{k}, Y)<\psi\left[g^{\prime}(\hat{k}), \hat{k}, Y\right]$. Since $(\partial \psi / \partial w)=\lambda-(s Y / \hat{k})$, if at some time $\tau, \dot{Y} \leqq 0$ and $Y<Y^{*}$, then $\dot{\hat{k}} \lessgtr 0 \Leftrightarrow w(\tau) \gtrless g^{\prime}[\hat{k}(\tau)] \Leftrightarrow \partial \psi / \partial w \lessgtr 0 \Leftrightarrow \lambda-(s Y \mid \hat{k}) \lessgtr 0 \Leftrightarrow \dot{w}(\tau) \gtrless 0$.

If $w(\tau)>g^{\prime}[\hat{k}(\tau)], \hat{k}$ must remain negative until $w(t)=g^{\prime}[\hat{k}(t)]<w(\tau)$, so $\dot{w}$ must become negative before $\dot{\hat{k}}$ can become positive. But if $\dot{w} \leqq 0$, $\dot{Y} \geqq 0$, by (4.14), since $\hat{k}(t)<\hat{k}(\tau)<s Y / \lambda<s Y^{*} / \lambda=k^{*}$. But this contradicts Lemma 1. Hence $\dot{w}(t)>0$ and $\dot{\hat{k}}(t)<0$ for all $t>\tau$, so $w(t)$ $-g^{\prime}[\hat{k}(t)]>0$ for all $t \geqq \tau$, that is, $\int_{A[\hat{k}(\tau), \tau]}\left\{w(v)-g^{\prime}[\hat{k}(\tau)]\right\} p(v, \tau) d v>0$. But this contradicts the perfect foresight assumption.

If $w(\tau)<g^{\prime}[\hat{k}(\tau)], \hat{k}$ must remain positive until $w(t)=g^{\prime}[\hat{k}(t)]>w(\tau)$, so $\dot{w}$ must become positive before $\dot{\hat{k}}$ can become negative. But by Lemma 1 , $\dot{Y} \mid Y \leqq 0 ;$ since $\hat{k}>0, \lambda-[s Y(t) \mid \hat{k}(t)]>\lambda-[s Y(\tau) \hat{k}(\tau)]>0$ so $\dot{w}(t)<0$ for all $t \geqq \tau$. But, again, this is inconsistent with perfect foresight.

Similarly, it is easy to establish some further qualitative properties of the behavior of income per capita. If $Y=Y^{*}$, but $\hat{k} \neq \hat{k}^{*}$, then $\dot{Y}>0$. If $Y=Y^{*}$ and $\hat{k}=\hat{k}^{*}$, then $\dot{Y}=0$. In Figure 6 we have reproduced the phase diagram from Part III. The curve $A E C$ is the locus of $\dot{Y}=0$ when $w=g^{\prime}(\hat{k})$, and $O E B$ is the locus of $\dot{w}=0$ when $w=g^{\prime}(\hat{k})$. Above

\footnotetext{
${ }^{14}$ If the derivative of $\dot{Y} / Y$ does not exist, we take the appropriate limit of the difference quotient.
} 


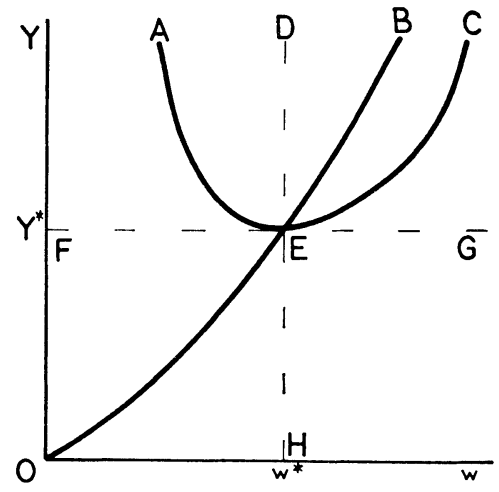

Fig. 6

$A E C, \dot{Y}<0$. To see this, observe that $\partial(\dot{Y} / Y) / \partial \hat{k}=s\left[w-g^{\prime}(\hat{k})\right] / \hat{k}^{2}$. Let $\tilde{k}$ be such that $w=g^{\prime}(\tilde{k})$. If $\hat{k}=\tilde{k}$, we have already established $\dot{Y}<0$, in Part III. If $\hat{k}>\tilde{k}$, that is, $w<g^{\prime}(\hat{k})$, we can write

$$
\psi(w, \hat{k}, Y)=\psi(w, \tilde{k}, Y)+\int_{\tilde{k}}^{\hat{k}} \frac{\partial \psi(w, k, Y)}{\partial k} d k<0 .
$$

Similarly, for $\hat{k}<\tilde{k}$.

We now establish the usual result:

\section{Lemma 3}

$$
\limsup _{t \rightarrow \infty} Y \leqq Y^{*} \cdot Y=\int_{\mathbf{k}}^{\infty} f(k) d N(k, t) \leqq \int_{0}^{\infty} f(k) d N(k, t) \leqq f(K)
$$

(by concavity), where

$$
K=\int_{0}^{\infty} k d N(k, t) .
$$

But $\dot{K}=-\lambda K+s Y \leqq-\lambda K+s f(K)$ so $\limsup _{t \rightarrow \infty} K \leqq K^{*}$ where $s f\left(K^{*}\right)=$ $\lambda K^{*}$, so $\limsup _{t \rightarrow \infty} Y \leqq f\left(K^{*}\right)=\lambda K^{*} / s=Y^{*}$.

Our stability theorems follow almost immediately.

\section{Theorem 4.1}

$$
\operatorname{Lim}_{t \rightarrow \infty} Y=Y^{*} .
$$

Either there exists a time $t$ at which $Y>Y^{*}$, or there does not. If there does not, since $\dot{Y}>0$ for $Y<Y^{*}, Y$ is monotonic and bounded, and thus must approach a limit. But again, since $\dot{Y}>0$ for $Y<Y^{*}$, the limit must 
be $Y^{*}$. If at some time $Y>Y^{*}$, by Lemmas 1 and 3, there must be a time after which $\dot{Y}$ is always negative. But by Lemma 2, if $Y$ is ever greater than $Y^{*}$, it can never be less than $Y^{*}$. Since $Y$ is thus bounded below by $Y^{*}$, and is monotonically decreasing after some time, it must approach a limit, greater than or equal to $Y^{*}$. But by Lemma $3, \limsup _{t \rightarrow \infty} Y \leqq Y^{*}$, so

$$
\lim _{t \rightarrow \infty} Y=Y^{*} .
$$

Theorem 4.2

$$
\operatorname{Lim}_{t \rightarrow \infty} \hat{k}=\hat{k}^{*}, \lim _{t \rightarrow \infty} w=w^{*} .
$$

Suppose $\lim _{t \rightarrow \infty} N(k, t) \neq 1$ for $k>\hat{k}^{*}, 0$ for $k \leqq \hat{k}^{*}$. Then, since $f(k)$ is strictly concave, for some $\epsilon>0$ and all $T<\infty$, there is a $t \geqq T$ such that $Y(t) \leqq f\left(K^{*}\right)-\epsilon$, again in contradiction to Theorem 4.1. Hence,

$$
\lim _{t \rightarrow \infty} \hat{k}=\hat{k}^{*} \text {. }
$$

But since by assumption $\hat{k}$ is continuous, if

$$
\lim _{t \rightarrow \infty} \hat{k}=\hat{k}^{*},
$$

by $(4.12)$, then

$$
\lim _{t \rightarrow \infty} w=w^{*} .
$$

We now establish some properties about the movements in $\hat{k}(t)$, the type of machine constructed at time $t$.

\section{Property 4.1}

In the region above $O E D$ (see phase diagram) $g^{\prime}(\hat{k}) \geqq w$, that is, $\dot{\hat{k}} \geqq 0$; in the region below $H E B, g^{\prime}(\hat{k}) \leqq w$, that is $\dot{\hat{k}} \leqq 0$. Consider the region above $O E D$. Assume that there existed a time $\tau$ at which $g^{\prime}[\hat{k}(\tau)]<w(\tau)$. Then for all $t>\tau, g^{\prime}[\hat{k}(t)]<w(t)$. In $O E D$, if $g^{\prime}(\hat{k})<w, \dot{w}>0$. Since $\dot{\hat{k}}(\tau)<0$, for $\dot{\hat{k}}$ to become non-negative there must exist a $t$ for which $\dot{w}$ must become negative before $\dot{\hat{k}}$ can become positive. But if $\dot{w}=0, \dot{Y}>0$, since $\hat{k}(t)<\hat{k}(\tau)<g^{-1}[w(\tau)] \leqq \hat{k}^{*}$ (and $\dot{Y} / Y=[s f(\hat{k}) / \hat{k}]-\lambda$ if $\left.\dot{w}=0\right)$; since $Y$ is increasing and $\hat{k}$ decreasing $\ddot{w}>0$. So $\dot{w} \geqq 0$ always. But then

$$
\int_{A[\hat{k}(\tau), \tau]}\left\{g^{\prime}[\hat{k}(\tau)]-w(v)\right\} p(v, \tau) d v<0,
$$

contrary to the perfect foresight profit maximization assumption. 
The other case may be handled in exactly the same manner. An immediate consequence of this is that the economy can only cross the line $O E$ from the right and below, and $B E$ from the left and above. Assume it crossed $O E$ from the left. When it reached $O E$, at a wage of $\tilde{w}, \hat{k} \geqq \tilde{k}$, where $g^{\prime}(\tilde{k})=\tilde{w}$. But that would mean that $\dot{w} \leqq 0$, and since $\dot{Y}>0$, that would mean that the trajectory crosses back into the region to the left of $O E$. Similarly for $B E$.

Similarly, we establish the following properties of wage movements.

\section{Property 4.2}

In $O E D$, if $\hat{k}>\hat{k}^{*}, \dot{w}>0$; in $B E H$, if $\hat{k}<\hat{k}^{*}, \dot{w}<0$. In $O E D$ assume at some time $\tau, \hat{k}(\tau): \hat{k}^{*}$, and $\dot{w}(\tau) \leqq 0$. Then $w$ falls forever. Assume $\dot{w}=0$. Then $\dot{Y}<0, \dot{\hat{k}}>0$, so $\ddot{w}(t)<0$. But this means that

$$
\int_{A[k(\tau), \tau]}\left\{w-g^{\prime}[k(\tau)]\right\} p(v, \tau) d v \neq 0,
$$

violating the perfect foresight profit maximization assumption. Similarly for $B E H$.

\section{Property 4.3}

In $F E D, \dot{w}>0$. In $G E H, \dot{w}<0$.

In $F E D$, if $\hat{k}>\hat{k}^{*}, \dot{w}>0$ by Property 4.2 . If $\hat{k} \leqq \hat{k}^{*},-\lambda+(s Y \mid \hat{k})>$ $-\lambda+\left(s Y^{*} / \hat{k}^{*}\right)=0$, so $\dot{w}>0$. Similarly for $G E H$.

With this information we can delineate five possible kinds of trajectories: (1) An economy can begin with $\dot{\hat{k}}<0$ (below $H E G$ ); at some time switch from $\dot{\hat{k}}<0$ to $\hat{k}>0$ (in $O E H$ ), cross $O E$, and switch back again to $\dot{\hat{k}}<0$ after crossing $D E$. (2) An economy can begin with $\dot{\hat{k}}<0$ and switch to $\dot{\hat{k}}>0$, as in (1), but never switch back. (3) An economy can begin with $\dot{\hat{k}}<0$ and never switch. (4) An economy can begin with $\dot{\hat{k}}>0$ and switch as in (1) to $\dot{\hat{k}}<0$. (5) An economy can begin with $\dot{\hat{k}}>0$ and never switch. (See Fig. 7.)

\section{Discontinuities in $\hat{\mathrm{k}}(\mathrm{t})$ and Construction of Many Types of Machines}

In the previous discussion we explicitly assumed that there was at any moment of time a single type of machine whose demand price was greater than or equal to that for all other kinds of machines. This assumption is likely to be satisfied for paths near balanced growth, since then any machine constructed can be used forever, and it is only obsolescence that results in the multiplicity of $\hat{k}$. But this assumption is not satisfied on all paths. Fortunately, most of the previous analysis carries through with only 

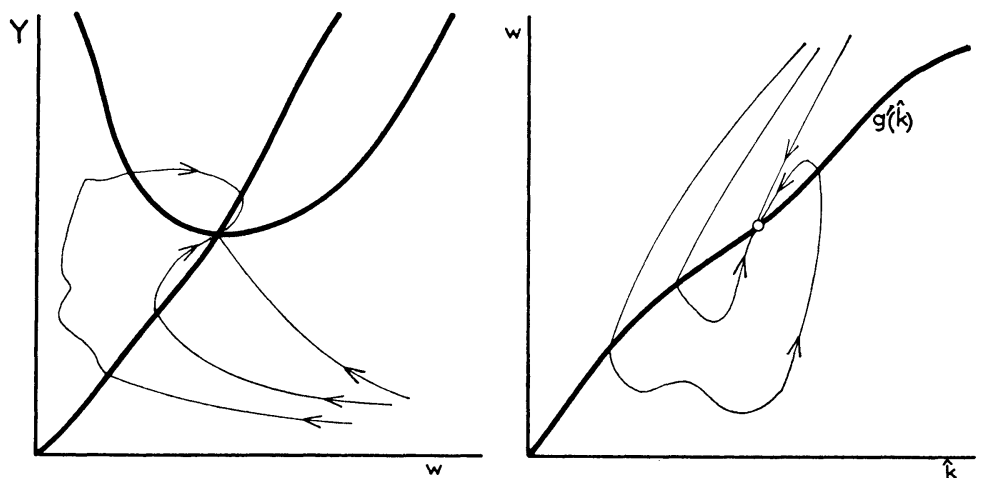

FIG. 7

slight modification. Again, our strategy will be to discover certain qualitative properties of the system which will not only provide us with information about the economy's evolution over time but will also enable us to prove convergence to the balanced growth path.

First, we prove that over any interval of time at most two "kinds" (in the sense defined below) of machines may be produced simultaneously. We define $\hat{k}_{1}(\tau)$ and $\hat{k}_{2}(\tau)$ by

$$
\begin{aligned}
& \hat{k}_{1}(\tau)=\min \left[k \mid \frac{f(k)-w}{k}=\frac{f(\tilde{k})-w}{\tilde{k}}\right], \\
& \hat{k}_{2}(\tau)=\max \left[k \mid \frac{f(k)-w}{k}=\frac{f(\tilde{k})-w}{\tilde{k}}\right],
\end{aligned}
$$

where $\tilde{k}=\min [k \mid q(k, \tau)=1]$; that is, we define the two types of machines with quasi-rent equal to the quasi-rent on the least capitalintensive machine with price 1 . In particular this entails $g^{\prime}\left(\hat{k}_{1}\right)<w<g^{\prime}\left(\hat{k}_{2}\right)$ when $\hat{k}_{1} \neq \hat{k}_{2}$. (See Fig. 8.) Since

$$
\frac{\dot{q}(k, t)}{q(k, t)}-\frac{\dot{q}(\hat{k}, t)}{q(\hat{k}, t)}=\frac{f(\hat{k})-w}{\hat{k} q(\hat{k}, t)}-\frac{f(k)-w}{k q(k, t)},
$$

the only types of machines that can be constructed at $t+\Delta t$ ( $\Delta t$ arbitrarily small) are $\hat{k} \leqq \hat{k}_{1}$ and $\hat{k} \geqq \hat{k}_{2}$. It immediately follows that if we produce at time $t$ two kinds of machines $\hat{k}_{1}(t)$ and $\hat{k}_{2}(t)$, and over an interval $(t, t+\Delta t)$ we produce two kinds, such that for any $\tau$ in the interval

$$
\lim _{\Delta \tau \rightarrow 0}\left|\hat{k}_{i}(\tau+\Delta \tau)-\hat{k}_{i}(\tau)\right|=0, \quad i=1,2,
$$




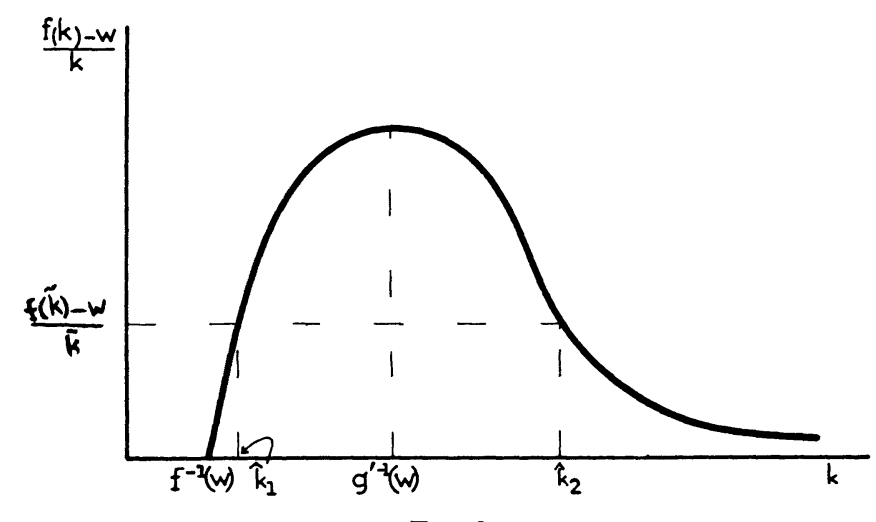

FIG. 8

then

$$
\frac{f\left[\hat{k}_{1}(\tau)\right]-w(\tau)}{\hat{k}_{1}(\tau)}=\frac{f_{2}\left(\hat{k}_{2}(\tau)\right]-w(\tau)}{\hat{k}_{2}(\tau)}
$$

for all $\tau$ in the interval.

We shall focus in the rest of the discussion on the "normal" cases where no machines of type $k \geqq \hat{k}^{*}$ exist initially, and therefore $Y<Y^{*}$, and $w<w^{*}$. (It should be clear, however, that the method of analysis is equally applicable to other initial conditions.)

Before proving that the system is stable, we note the following important relations between $\hat{k}_{1}, \hat{k}_{2}$, and $\dot{w}:^{15}$

$$
\begin{aligned}
& \text { If } \dot{\hat{k}}_{2}<0 \text { and } \dot{w}>0 \text {, then } \dot{\hat{k}}_{1}>0 . \\
& \text { If } \dot{\hat{k}}_{2}>0 \text { and } \dot{w}<0 \text {, then } \dot{\hat{k}}_{1}<0 . \\
& \text { If } \dot{\hat{k}}_{1}<0 \text { and } \dot{w}>0 \text {, then } \dot{\hat{k}}_{2}>0 . \\
& \text { If } \dot{\hat{k}}_{1}>0 \text { and } \dot{w}<0 \text {, then } \dot{\hat{k}}_{2}<0 .
\end{aligned}
$$

We now show that if $Y \leqq Y^{*}, w$ cannot become greater than $w^{*}$. Assume at some time $\tau_{0}, Y\left(\tau_{0}\right)<Y^{*}$ and $w\left(\tau_{0}\right)=w^{*}$. If $\dot{w}\left(\tau_{0}\right)>0, \hat{k}_{1}\left(\tau_{0}\right)<\hat{k}^{*}$, then $\hat{k}_{2}\left(\tau_{0}\right)>\hat{k}^{*}$. For $\dot{w}$ to remain positive, $\hat{k}_{1}$ must be constructed, so $\dot{\hat{k}}_{1}$ $<0$ and $\hat{k}_{2}>0$. Eventually $w$ must return to less than $w^{*}$, since otherwise

$$
\int_{A(k, t)}\left[w-g^{\prime}\left(\hat{k}_{1}\right)\right] p(v, t) d v>0 .
$$

For $\dot{w}$ to become negative, either:

a) $Y \geqq Y^{*}$, and $\hat{k}_{2}$ is constructed, $\dot{\hat{k}}_{2}>0$, and $\dot{\hat{k}}_{1}<0$; or

${ }^{15}$ Throughout this section, $\dot{\hat{k}}_{\mathfrak{t}}<0$ is to be read $\hat{k}_{\mathfrak{l}}$ is decreasing, etc., not necessarily implying that $\hat{k}_{i}(t)$ is differentiable. 
b) $Y<Y^{*}, \dot{Y} \geqq 0$. Since up to the time that $\dot{w}<0, \hat{k}_{1}$ was constructed, $\dot{\hat{k}}_{1}<0$; for $\dot{w}<0, \hat{k}_{2}$ must be constructed, $\dot{\hat{k}}_{2}>0$, and $\dot{\hat{k}}_{1}<0$; or

c) $Y<Y^{*}, \dot{Y}<0 .{ }^{16}$ If $\hat{k}_{1}$ only were constructed, for $\dot{Y}<0, \dot{w}>0$.

So again $\hat{k}_{2}$ is constructed, $\dot{\hat{k}}_{2}>0$, and $\dot{\hat{k}}_{1}<0$.

Since $\hat{k}_{2}$ is being constructed as $w$ returns to the value $g^{\prime}\left[\hat{k}_{1}\left(\tau_{0}\right)\right]<w^{*}$ and $\hat{k}_{2}>\hat{k}^{*}$, we must again eventually exceed $w^{*}$. But for $\dot{w}$ to be positive, either:

a) $Y \leqq Y^{*}$, and $\hat{k}_{1}$ is constructed, so $\dot{\hat{k}}_{1}<0, \dot{\hat{k}}_{2}>0$; or

b) $Y>Y^{*}, \dot{Y} \leqq 0$. Since up to the time that $\dot{w}>0, \hat{k}_{2}$ was constructed, and $\dot{\hat{k}}_{2}>0$, for $\dot{w}>0, \hat{k}_{1}$ must be constructed, $\dot{\hat{k}}_{2}>0$, and $\dot{\hat{k}}_{1}<0$; or

c) $Y<Y^{*}, \dot{Y}>0$. If $\hat{k}_{2}$ only were constructed, for $\dot{Y}>0, \dot{w}<0$.

So again $\hat{k}_{1}$ is constructed, $\dot{\hat{k}}_{2}>0$, and $\dot{\hat{k}}_{1}<0$.

Thus, $\dot{w}$ must continue to oscillate around $w^{*}$; as it does this $\hat{k}_{2}$ rises and $\hat{k}_{1}$ falls. Moreover, since at least one of these is changing at a rate bounded away from zero, for some finite $\tilde{\tau}>\tau_{0}, g^{\prime}\left[\hat{k}_{2}(\tilde{\tau})\right]>f\left[\hat{k}_{1}(\tilde{\tau})\right]>w(\tilde{\tau})$. But that means that the economy's wage cannot exceed $f\left[\hat{k}_{1}(\tilde{\tau})\right]$, since to do so requires the construction of $\hat{k}_{1}(\tilde{\tau})$, but since no new machines of type $\hat{k}_{1}(\tilde{\tau})$ are added to production, $\dot{w}(\tilde{\tau})<0$. But this means

$$
\int_{A\left(k_{2}, t\right)}\left[w-g^{\prime}\left(\hat{k}_{2}\right)\right] p(v, t) d v \neq 0,
$$

for some $\hat{k}_{2}$ constructed previously, so this could not have been a perfect foresight path.

Similarly it can be shown that $Y \leqq Y^{*}$; for $Y$ to be greater than $Y^{*}$, at some time the $\hat{k}$ constructed must be greater than $\hat{k}^{*}$. Since $w \leqq w^{*}$, $\hat{k}_{1}<\hat{k}^{*}$, and the analysis proceeds as above.

Convergence of $Y$ to $Y^{*}$ is now easy to establish. If $Y<Y^{*}, \dot{Y}$ cannot be negative, since that would imply that, if $\hat{k}_{1}$ alone were constructed, $\dot{w}$ $>0$; if $\hat{k}_{2}$ alone were constructed, $\dot{w}<0$. But, as earlier, this leads to an inconsistency. ${ }^{17}$ Since $Y \leqq Y^{*}, Y$ must then converge to $Y^{*}$. But since the type of machine constructed is less than or equal to $\hat{k}^{*}$, for $Y$ to converge to $Y^{*}, \hat{k}$ must converge to $\hat{k}^{*}$, and $w$ must converge to $w^{*}$.

${ }^{16}$ As before, it is possible to show that if $\dot{Y} \leqq 0$ ever, it is always less than or equal to zero. $\dot{Y}$ is not defined only if $\dot{w}$ is not defined; see above, Part II.

${ }^{17}$ For $\hat{k}_{1}$ continues to fall, $\hat{k}_{2}$ continues to rise; eventually either $g^{\prime}\left(\hat{k}_{2}\right)>w^{*}$, or $f\left(\hat{k}_{1}\right)<g^{\prime}\left(\hat{k}_{2}\right)$. 


\section{Stability of the Pricing System}

A great deal of attention has been focused recently on the instability of the pricing system in growth models with heterogeneous capital goods. In particular, Hahn (1966) has shown that under some simple saving assumptions and short-run perfect foresight with perfect capital markets (that is, where all capital goods yield the same rate of return) for any given initial capital endowments, not all initial price vectors lead to balanced growth. This result was strengthened by Shell and Stiglitz (1967) who showed in the context of a very simple model that there was a unique set of initial prices which could lead to balanced growth. But they then pointed out that for all other initial prices, at some finite time in the future, the perfect foresight assumption would be violated. The results of this section support that result: there are initial price systems (that is, $q(k, 0)$ ) for which the economy does not converge to balanced growth (for simple initial employment distributions, for all but one). However, these are inconsistent with perfect foresight holding at every moment of time. To put it another way, all equilibrium paths must converge to balanced growth.

\section{Optimal Saving ${ }^{18}$}

Thus far we have assumed that the economy is composed of decentralized decision makers. Suppose now that all decision making is centralized in the hands of a planning authority whose objective is to allocate final output between consumption and investment, and investment over the available techniques, to maximize the discounted stream of future consumption per capita:

$$
\int_{0}^{\infty} C(t) e^{-\delta t} d t \text { with } \delta>0
$$

subject to a subsistence level constraint

$$
0<\mathbf{C} \leqq C(t) \leqq Y(t) .{ }^{19}
$$

${ }^{18}$ A much more complete investigation of the sort of model sketched in this section is being carried out by Calvo (1967).

${ }^{19}$ This objective can be rewritten as maximize

where

$$
\int_{0}^{\infty} U[C(t)] e^{-\delta t} d t \text { with } \delta>0,
$$

$$
U(C)\left\{\begin{aligned}
=-\infty, & \text { for } \quad C<\mathbf{C} \\
=C, & \text { for } \quad C \geqq \mathbf{C} .
\end{aligned}\right.
$$

Such an instantaneous utility function is one approximation to the general instantaneous utility function satisfying $U^{\prime}(C)>0$ with

$$
\lim _{C \rightarrow 0} U^{\prime}(C)=\infty \quad \text { and } \quad U^{\prime \prime}(C) \leqq 0 .
$$


As might be expected, the path chosen by the planning authority is closely related to the perfect foresight paths analyzed in Part IV, but with a major difference: not only must the choice of technique be profit maximizing for a certain system of prices, aggregate saving must be output-value maximizing at those same prices.

To be more precise, let $p(t)$ now represent the value of a unit of investment at time $t$ in terms of consumption per capita at time 0 or, more simply, the present value of a unit of investment at time $t$ (so that $\left[p(t) e^{\delta t}\right]$ represents the value of a unit of investment at time $t$ in terms of consumption per capita at time $t$, or, simply, the value of a unit of investment at time $t)$. Notice that $p(t)\left[p(t) e^{\delta t}\right]$ need not equal $e^{-\delta t}[1]$, the present value [value] of a unit of consumption per capita at time $t$. Moreover, it should be clear that the present value [value] of a unit of final output at time $t$ is

$$
\tilde{p}(t)=\max \left[e^{-\delta t}, p(t)\right]\left\{\tilde{p}(t) e^{\delta t}=\max \left[1, p(t) e^{\delta t}\right]\right\} .
$$

Finally, let $\tilde{p}(t) w(t)\left[\tilde{p}(t) w(t) e^{\delta t}\right]$ be the present value [value] of a unit of labor at time $t$.

Now, for any given system of these prices we can define the present value [value] of a machine of type $k$ at time $t$, analogously to (4.4), by

$$
q(k, t) e^{-\delta t}=\int_{A(k, t)} \tilde{p}(v) \frac{f(k)-w(v)}{k} e^{-\lambda(v-t)} d v .^{20}
$$

Also, given any such system of demand prices $q(k, t)$, for a given feasible ${ }^{21}$ path, say, denoted prime, we can define the value of the capital stock at time $t$ by

$$
V^{\prime}(t)=\int_{0}^{\infty} q(k, t) k d N^{\prime}(k, t)
$$

From an argument parallel to that justifying (2.8), we can show that the value $V^{\prime}(t)$ evolves according to the equation: $:^{22}$

$$
\dot{V}^{\prime}(t)-\delta V^{\prime}(t)=q\left[\hat{k}^{\prime}(t), t\right] I^{\prime}(t)-\tilde{p}(t) e^{\delta t} \int_{f^{-1}[w(t)]}^{\infty}[f(k)-w(t)] d N^{\prime}(k, t) .
$$

${ }^{20}$ The reason $\lambda$ rather than $\mu$ appears in this definition is that consumption per capita at time 0 (time $t)$ is the numeraire.

21 "Feasible" is taken to mean behavior which satisfies the full employment and output equations (2.1) and (2.3) for some specification of aggregate saving and choice of technique given the initial employment distribution function.

${ }^{22}$ For ease of notation, which is bad enough as is, we assume that at each instant there is only one type of machine $\hat{k}^{\prime}(t)$ being built; the argument when there are a finite number of types of machines being built is a straightforward generalization. While even the latter is not as general as permitted by the underlying technology, it is certainly broad enough to encompass the interesting economic implications of such a technology. 
Along an optimal path (5.5) simply says that change in the value of the capital stock is equal to investment plus capital gains (see also the discussion of eq. 7.7 below).

With these preliminary definitions in hand we can characterize the behavior induced by the planning authority in two propositions: the first states sufficient conditions for optimal saving behavior; the second describes (at least in a neighborhood of balanced growth) actual behavior satisfying these conditions.

\section{Theorem 5.1}

Suppose we can find prices $\mathrm{p} *(\mathrm{t})$ and $\mathrm{w} *(\mathrm{t})$ and a feasible path, also denoted asterisk, which satisfy the following conditions:

i) $\mathrm{C}^{*}(\mathrm{t})$ maximizes $\mathrm{C}(\mathrm{t})+\mathrm{p}^{*}(\mathrm{t}) \mathrm{e}^{\delta \mathrm{t}}\left[\mathrm{Y}^{*}(\mathrm{t})-\mathrm{C}(\mathrm{t})\right]$ subject to $\mathrm{C} \leqq \mathrm{C}(\mathrm{t}) \leqq \mathrm{Y}^{*}(\mathrm{t})$

ii) $\mathrm{f}\left[\mathrm{k}^{*}(\mathrm{t})\right]=\mathrm{w}^{*}(\mathrm{t})$;

iii) $\mathrm{q}^{*}(\mathrm{k}, \mathrm{t}) \leqq \mathrm{p}^{*}(\mathrm{t}) \mathrm{e}^{\delta \mathrm{t}}$ with equality for $\mathrm{k}=\hat{\mathrm{k}}^{*}(\mathrm{t})$; and

iv) $\lim _{\mathrm{t} \rightarrow \infty} \mathrm{V}^{*}(\mathrm{t}) \mathrm{e}^{-\delta \mathrm{t}}=0$.

Then this path maximizes (5.1) subject to (5.2).

Note that the theorem goes through for a variable discount rate $\delta(t)$ as well. From this fact it is easily argued that along the perfect foresight paths of Part IV, if $s$ is low enough so that $f^{\prime}\left(\hat{k}^{*}\right)-\lambda>0$, that is, the long-run private rate of return is greater than the population growth rate, then the social rate of return on investment is equal to the private rate of return, that is, the perfect foresight path maximizes

$$
\int_{0}^{\infty} p(t) C(t) d t
$$

where $p(t)$ is again given by (4.2). (The border-line cases $\delta=0$ and $f^{\prime}\left(k^{*}\right)$ $-\lambda=0$ can also be included if one adopts the "Ramsey device" of Koopmans [1965] or the "overtaking criterion" of von Weizsäcker [1965].) A similar point was made by Solow, Tobin, von Weizsäcker, and Yaari (1966) for the vintage model with ex ante rigid complementarity but Harrod neutral embodied technical progress.

\section{Proof}

The first condition of the theorem may be rewritten:

$$
\begin{aligned}
& \text { if } p^{*}(t)<e^{-\delta t}, \text { then } C^{*}(t)=Y^{*}(t) \\
& \text { if } p^{*}(t)=e^{-\delta t}, \text { then } \mathbf{C} \leqq C^{*}(t) \leqq Y^{*}(t) ; \\
& \text { if } p^{*}(t)>e^{-\delta t}, \text { then } \mathbf{C}=C^{*}(t) .
\end{aligned}
$$


Now consider the evaluation of any feasible path satisfying the subsistence level constraint (5.2) given by the objective function (5.1):

$$
\begin{aligned}
\int_{0}^{\infty} C^{\prime}(t) e^{-\delta t}= & \int_{0}^{\infty}\left\{C^{\prime}(t) e^{-\delta t}+\tilde{p}^{*}(t)\left[\int_{\mathbf{k}^{\prime}(t)}^{\infty} f(k) d N^{\prime}(k, t)-Y^{\prime}(t)\right]\right. \\
& \left.\quad+\tilde{p}^{*}(t) w^{*}(t)\left[1-\int_{\mathbf{k}^{\prime}(t)}^{\infty} d N^{\prime}(k, t)\right]\right\} d t \\
= & \int_{0}^{\infty}\left[e^{-\delta t}-\tilde{p}^{*}(t)\right] C^{\prime}(t) d t+\int_{0}^{\infty} \tilde{p}^{*}(t) \\
& \times\left\{\int_{\mathbf{k}^{\prime}(t)}^{\infty}\left[f(k)-w^{*}(t)\right] d N^{\prime}(k, t)-I^{\prime}(t)\right\} d t \\
& +\int_{0}^{\infty} \tilde{p}^{*}(t) w^{*}(t) d t \\
= & \int_{0}^{\infty}\left[e^{-\delta t}-\tilde{p}^{*}(t)\right] C^{\prime}(t) d t \\
& +\int_{0}^{\infty} \tilde{p}^{*}(t) \int_{\mathbf{k}^{\prime}(t)}^{f^{-1}\left[w^{*}(t)\right]}\left[f(k)-w^{*}(t)\right] d N^{\prime}(k, t) d t \\
& +\int_{0}^{\infty}\left\{q^{*}\left[\hat{k}^{\prime}(t), t\right] e^{-\delta t}-\tilde{p}^{*}(t)\right\} I^{\prime}(t) d t \\
& +\int_{0}^{\infty}\left\{\tilde{p}^{*}(t) \int_{f^{-1}\left[w^{*}(t)\right]}^{\infty}\left[f(k)-w^{*}(t)\right] d N^{\prime}(k, t)\right. \\
& +\int_{0}^{\infty} \tilde{p}^{*}(t) w^{*}(t) d t, \\
& \left.-q^{*}\left[\hat{k}^{\prime}(t), t\right] e^{-\delta t} I^{\prime}(t)\right\} d t
\end{aligned}
$$

(utilizing [5.5] to simplify the fourth term of the preceding expression),

$$
\begin{aligned}
& =\int_{0}^{\infty}\left[e^{-\delta t}-\tilde{p}^{*}(t)\right] C^{\prime}(t) d t \\
& +\left\{\int_{0}^{\infty} \tilde{p}^{*}(t) \int_{\mathbf{k}^{\prime}(t)}^{f^{-1}\left[w^{*}(t)\right]}\left[f(k)-w^{*}(t)\right] d N^{\prime}(k, t) d t\right\} \\
& +\int_{0}^{\infty}\left\{q^{*}\left[\hat{k}^{\prime}(t), t\right] e^{-\delta t}-p^{*}(t)\right\} I^{\prime}(t) d t \\
& -\left[V^{\prime}(t) e^{-\delta t}\right]_{0}^{\infty}+\int_{0}^{\infty} \tilde{p}^{*}(t) w^{*}(t) d t .
\end{aligned}
$$


Analyzing the last expression term by term, we find that (i) or (5.6) implies that the first term is a maximum when $C^{\prime}(t)=C^{*}(t)$, (ii) implies the second is a maximum when $f\left[k^{\prime}(t)\right]=w^{*}(t)$, (iii) implies the third is a maximum when $\hat{k}^{\prime}(t)=\hat{k}^{*}(t)$, (iv) implies the fourth is a maximum when $V^{\prime}(t)=$ $V^{*}(t)$, while the fifth is identical on every feasible path. But this is precisely what the theorem asserts.

\section{Theorem 5.2}

Define the optimal balanced growth path by $\mathrm{f}^{\prime}\left(\hat{\mathrm{k}}^{*}\right)=\delta+\lambda, \mathrm{w}^{*}=\mathrm{f}\left(\hat{\mathrm{k}}^{*}\right)-$ $\mathrm{f}^{\prime}\left(\hat{\mathrm{k}}^{*}\right) \hat{\mathrm{k}}^{*}, \mathrm{Y}^{*}=\mathrm{f}\left(\hat{\mathrm{k}}^{*}\right)$ and $\mathrm{C}^{*}=\mathrm{Y}^{*}-\lambda \hat{\mathrm{k}}^{*}$. Assume that consumption on this path is greater than the subsistence level $\mathrm{C}^{*} \geqq \mathrm{C}$. If the initial ouput $\mathrm{Y}(0)$ and wage rate $\mathrm{w}(0)$ associated with a given initial employment distribution function $\mathrm{N}(\mathrm{k}, 0)$ are sufficiently close to $\mathrm{Y}^{*}$ and $\mathrm{w}^{*}$, then there exists a feasible path satisfying the conditions of Theorem 5.1 and exhibiting the following properties: for some $0 \leqq \mathrm{t}^{\prime}<\infty$ :

(i) $\mathrm{C}^{*}(\mathrm{t})=\mathbf{C}\left[\mathrm{Y}^{*}(\mathrm{t})\right]$, for $0 \leqq \mathrm{t}<\mathrm{t}^{\prime}$ when $\mathrm{w}(0)<\mathrm{w}^{*}\left[\mathrm{w}(0)>\mathrm{w}^{*}\right]$ $=\mathrm{Y}^{*}(\mathrm{t})-\lambda \hat{\mathrm{k}}^{*}$, for $\mathrm{t}^{\prime} \leqq \mathrm{t}$;

(ii) $\mathrm{w}^{*}(\mathrm{t})<\mathrm{w}^{*}\left[>\mathrm{w}^{*}\right]$, for $0 \leqq \mathrm{t}<\mathrm{t}^{\prime}$ when $\mathrm{w}(0)<\mathrm{w}^{*}\left[\mathrm{w}(0)>\mathrm{w}^{*}\right]$ $=\mathrm{w}^{*}$, for $\mathrm{t}^{\prime} \leqq \mathrm{t}$;

(iii) $\hat{\mathrm{k}}^{*}(\mathrm{t})<\hat{\mathrm{k}}^{*}\left[>\hat{\mathrm{k}}^{*}\right]$, for $0 \leqq \mathrm{t}<\mathrm{t}^{\prime}$ when $\mathrm{w}(0)<\mathrm{w}^{*}\left[\mathrm{w}(0)>\mathrm{w}^{*}\right]$ $=\hat{\mathrm{k}}^{*}$, for $\mathrm{t}^{\prime} \leqq \mathrm{t}$; and

(iv) $\lim _{\mathrm{t} \rightarrow \infty} \mathrm{Y}^{*}(\mathrm{t})=\mathrm{Y}^{*}, \lim _{\mathrm{t} \rightarrow \infty} \mathrm{C}^{*}(\mathrm{t})=\mathrm{C}^{*}$.

In terms of the now familiar phase diagram for the behavior of $Y$ and $w$, the two typical cases are illustrated in Figure 9.

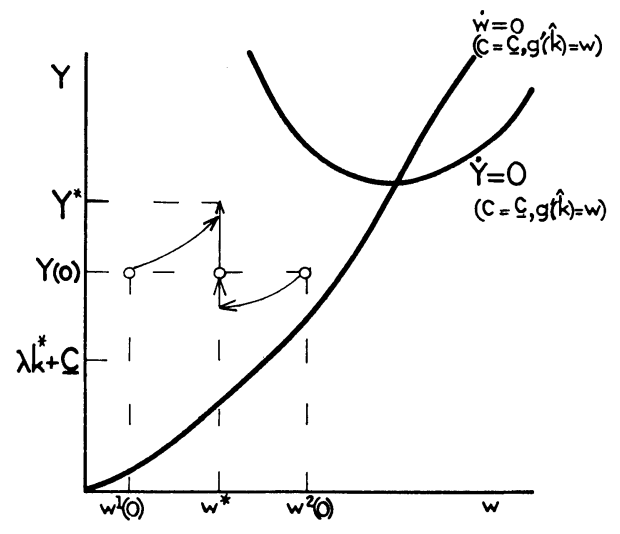

FIG. 9 
Proof $^{23}$

Because it is the most interesting (as in a sense it represents underdevelopment), we concentrate on the case where $w(0)<w^{*}$; the alternative case where $w(0)>w^{*}$ is essentially the same. The argument hinges on the assumption that $[Y(0), w(0)]$ is close to $\left(Y^{*}, w^{*}\right)$, since this assumption allows us to verify a provisional hypothesis that there is never obsolescence associated with the types of machine built after time 0 .

We observe first that if $Y(0) \geqq \lambda \hat{k}^{*}+\mathbf{C}$ and $w(0)=w^{*}$, then the path described in the theorem exists for $t^{\prime}=0$. The planning authority simply invests enough in machines of type $\hat{k}^{*}$ to maintain the wage rate $w^{*}$ and thereby provides consumption at least as great as the subsistence level, while the economy approaches the balanced growth path. Moreover, it is easily verified that this path satisfies the conditions of Theorem 1 with $p^{*}(t)=e^{-\delta t}$. This result implies that whenever the economy reaches a point at which $Y(t) \geqq \lambda \hat{k}^{*}+\mathbf{C}$ and $w(t)=w^{*}$, henceforth the planning authority should proceed precisely this same way. Thus, all we need show is that, starting close to $\left(Y^{*}, w^{*}\right)$, the planning authority can optimally maneuver to a point $Y\left(t^{\prime}\right) \geqq \lambda \hat{k}^{*}+\mathbf{C}$ and $w\left(t^{\prime}\right)=w^{*}$ at some time $t^{\prime}<\infty$.

To see that this is indeed possible, suppose that each type of machine which is built after time 0 is utilized forever. Then, being chosen according to perfect foresight, $\hat{k}$ must satisfy the appropriate form of equation (4.12), which is

$$
\dot{\hat{k}}=\frac{w-g^{\prime}(\hat{k})}{f^{\prime \prime}(\hat{k}) \hat{k} / f^{\prime}(\hat{k})} .
$$

Also assume that $C(t)=\mathbf{C}$. Under these hypotheses, if $w(0)$ is close to $w^{*}$ and $\hat{k}(0)$ is chosen near or in $\left\{g^{\prime^{-1}}[w(0)], \hat{k}^{*}\right\}$, then, at least initially, the behavior of $Y$ and $w$ is basically as it would be if $\hat{k}$ were chosen according to static expectations (such behavior depicted in Fig. 9 for $\mathbf{C}$ close to 0 , or $s=s_{\pi}=s_{w}$ variable but close to 1$)$. Hence, provided $Y(0)$ is sufficiently close to $Y^{*}, Y$ and $w$ are both increasing. But, in conjunction with equation (5.8), this means that by ranging $\hat{k}(0)$ from $\geqq \hat{k}^{*}$ to $\leqq g^{\prime-1}[w(0)]$ we must find a unique $g^{-1}[w(0)]<\hat{k}^{*}(0)<\hat{k}^{*}$ such that $w(t) \leq w^{*}$ and $\hat{k}(t) \leq \hat{k}^{*}$, according as $t \leq t^{\prime}$ for some $t^{\prime}<\infty$. (This argument is represented diagrammatically in Fig. 10.) Moreover, for $Y(0)$ sufficiently close to $Y^{*}$ we have $Y(t) \geqq \lambda \hat{k}^{*}+\mathbf{C}$, while for $w(0)$ sufficiently close to $w^{*}$ we have $w(t) \leqq w^{*}<f\left[\hat{k}^{*}(0)\right] \leqq f[\hat{k}(t)]$; the former simply says that at time $t^{\prime}$ the planning authority should start along the balanced growth path, the

${ }^{23}$ In this proof we implicitly assume that the initial employment distribution function satisfies a Lipschitz condition on an interval including $\left[k(0), k^{*}\right]$ or $\left[k^{*}, k(0)\right]$. This enables us to utilize standard results concerning the existence and continuity with respect to initial conditions of solutions to a system of differential equations; modification of the argument to accommodate isolated discontinuities in the initial distribution is relatively straightforward. 


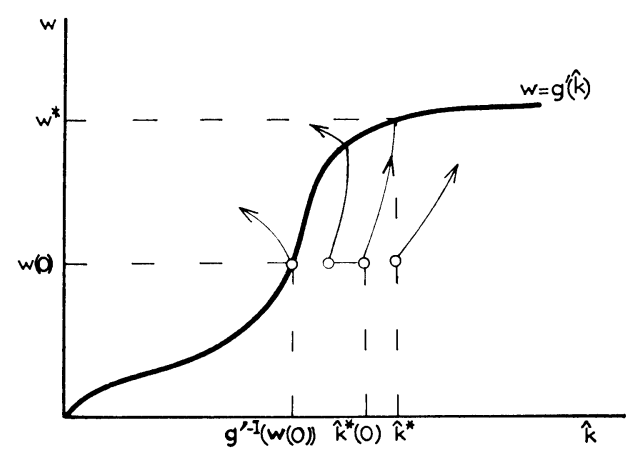

FIG. 10

latter that, provided the planning authority does, the types of machine built after time 0 will never become obsolete.

To complete the argument we must find a system of present values $p^{*}(t)$ for which, along the path just described, we have (1) the choice of technique (5.8) satisfying condition (iii) of Theorem 5.1, and (2) the capital stock (5.4) satisfying condition (iv) of Theorem 5.1. But direct calculation, the details of which are omitted here, verifies that

$$
\dot{p}^{*}=p^{*}\left[\lambda-\frac{f(\hat{k})-w}{\hat{k}}\right] \text { with } p^{*}\left(t^{\prime}\right)=e^{-\delta t^{\prime}}
$$

will do nicely. ${ }^{24}$ (Intuitively, [5.9] simply recognizes that the social rate of return had better equal the net quasi-rent from the type of machine being built currently.)

We strongly conjecture that, starting from an arbitrary initial employment distribution function, optimal saving behavior will exhibit, at least eventually, the properties outlined here. However, answers to the really interesting questions raised in a wider context, for example, the nature of the dependence of obsolescence or specialization (that is, consuming at the subsistence level or investing at a zero level) on the particular form of the initial distribution, must await fuller analysis. ${ }^{25}$

In any event, while the sufficient conditions for optimal saving behavior here are quite similar to those for the malleable capital model, the evolu-

${ }^{24}$ To satisfy condition (iii). However, if condition (iii) is satisfied and

$$
\lim _{t \rightarrow \infty} p^{*}(t)=0,
$$

then condition (iv) is also satisfied.

${ }^{25}$ We should mention that obsolescence is clearly possible even with optimal saving behavior; the very "underdeveloped" economy may adopt relatively labor intensive techniques today only to discard them in favor of relatively capital intensive techniques tomorrow. It is fairly easy to construct an example of this phenomenon when $\lambda=0$. 
tion of the economy near optimal balanced growth is quite different. On the one hand, the optimal saving rate is chosen to maximize the value of final output (condition [i]), while the present value of the capital stock goes to zero (condition [iv]), where both quantities are evaluated in terms appropriate to the objective of the planning authority (5.1). On the other hand, we notice that here the initial distribution of capital may entail both an earlier phase in which the economy's allocation of output is specialized and a later phase in which the economy's type of investment is specialized, where both phases have the effect of bringing the economy's production possibilities into accord with the planning authority's rate of time preference. In the malleable capital model, these two phases coincide.

\section{Probabilistic Expectations}

So far, we have considered two extremes in the formation of expectations: perfect foresight, where expectations are always realized, and static expectations, where expectations are never realized outside of steady states. What these two theories of expectations formation have in common is that the individual forms point estimates of wages and interest rates over the future and acts on those point estimates as if they were certain. It seems somewhat more reasonable to assume that individuals, when faced with uncertainty, recognize the existence of this uncertainty and act accordingly, that is, form some kind of probability distribution of outcomes and maximize the expectation of some functional of those outcomes. Indeed, this is the basic approach of modern portfolio theory and monetary theory. Should it not be equally applicable to the problems of the choice of technique? The following discussion should be considered only a first, tentative approach to these problems, but hopefully it will suggest lines along which future research may be directed.

Consider an individual with a given wealth which he wishes to allocate over a portfolio of capital goods. Presumably, he wishes to do this in such a way as to maximize his expected (intertemporal) utility. In practice, it is more likely that he follows some simplified procedure, such as maximizing expected utility of the return on the portfolio or maximizing expected utility of wealth over some period. We shall follow the usual portfolio analysis of Tobin (1958), for example, and assume the portfolio of capital goods is chosen to maximize $E U(R)$ where $E$ is the expectation operator,

$$
R=\int_{0}^{\infty}\left[\frac{f(k)-w}{k q(k, t)}+\frac{\dot{q}(k, t)}{q(k, t)}\right] a(k) d k=\int_{0}^{\infty} r(k, t) a(k) d k,
$$

and $a(k)$ is the proportion of one's portfolio invested in capital of type $k$, so that

$$
\int_{0}^{\infty} a(k)=1
$$


The necessary conditions for the optimal allocation can be written in parametric form:

$$
\begin{gathered}
E U^{\prime} r(k, t) \leqq \eta ; \\
a(k)\left[E U^{\prime} r(k, t)-\eta\right]=0 .
\end{gathered}
$$

The expected marginal utility of all capital goods held in the portfolio must be the same.

The first question we ask is: how many of the capital goods held in the portfolio are simultaneously being produced, that is, have a price of unity? We shall assume that the individual acts as if prices are differentiable (that is, $\dot{q}[k, t]$ exists), so for a capital good being produced, $\dot{q}=0$. Thus, we are asking: for how many capital goods can

$$
E U^{\prime} \frac{f-w}{k}=\eta ?
$$

As before, the expectation is taken only over those states of nature where $f>w$. But if the range of possible wages is sufficiently small, the only capital intensities that need to be considered are those for which the machines will be used in all states of nature. In particular, if

$$
w_{\min }^{e}
$$

is the minimum expected wage, and

$$
w_{\max }^{e}
$$

is the maximum, then we shall assume that

$$
f\left[g^{\prime-1}\left(w_{\min }^{e}\right)\right] \geqq w_{\max }^{e} .
$$

If, for instance, the ex ante production function is Cobb-Douglas with capital exponent of $\frac{1}{4}$, then the range of uncertainty on the wage can be as large as $33 \frac{1}{3}$ per cent.

Under this assumption, we can take the derivative of the left-hand side of equation (6.5) with respect to $k$ and obtain

$$
\frac{\partial\left[E U^{\prime} \frac{f-w}{k}\right]}{\partial k}=E U^{\prime}\left[\frac{w-g^{\prime}(k)}{k^{2}}\right],
$$

for $k$ in the relevant range.

There is a unique point at which the left-hand side of equation (6.5) is maximized, since then $E\left\{U^{\prime}\left[w-g^{\prime}(\hat{k})\right]\right\}=0$, and this expression is unambiguously decreasing in $k$, that is, $-E U^{\prime} g^{\prime \prime}(\hat{k})<0$. Thus, any given individual will hold at most one type of new capital good in his portfolio.

How does his choice of the type of capital constructed differ from his 
choice if he had acted on the point estimate of the mean? We wish to compare $g^{\prime}(\hat{k})=\left(E U^{\prime} w / E U^{\prime}\right)$ with $g^{\prime}(\tilde{k})=E w$.

So far, the analysis has been fairly general. To answer this question, however, we must know how the prices of the different kinds of capital goods are determined and how they change over time. We can simplify the analysis by assuming that there are no markets for used capital goods, so that an individual purchases the machines only for the quasi-rents he can obtain from them, not for short-term capital gains. Then $r(k, t)=$ $[f(k)-w(t)] / k$, a decreasing function of $w$. Thus $E\left(U^{\prime}-\bar{U}^{\prime}\right)(w-\bar{w})$ $>0$. But $\hat{k}>\tilde{k}$ if and only if $g^{\prime}(\hat{k})>g^{\prime}(\tilde{k})$ if and only if $E U^{\prime} w / E U^{\prime}>E w$ if and only if $E\left(U^{\prime}-\bar{U}^{\prime}\right)(w-\bar{w})>0$, that is, the covariance between marginal utility and the wage is positive. Hence, we have shown that uncertainty raises the capital intensity of the machine constructed.

A further difficulty is now introduced into the dynamic analysis. The loci of $(\dot{w}=0, \dot{Y}=0)$ may depend on the exact nature of the distribution of wages. In the following special case, we can establish local stability in much the same manner as in the previous cases.

For convenience, we assume that the degree of uncertainty (for example, the variance of wage expectation) is a function of the amount of change in the economy, for example, $\dot{w} / w$, with zero variance if $\dot{w} / w=0$, and that the mean wage is the wage today. Assuming again that a constant fraction of income is saved, along the curve $s Y / g^{\prime-1}(w)=\lambda, \dot{w}=0$, to the left of it $\dot{w}>0$, to the right of it $\dot{w}<0$. Although the exact slope or position of the $\dot{Y}=0$ curve cannot be determined (and indeed may shift over time), it lies everywhere above $Y=Y^{*}$, and it is easy to show, consequently, that $Y$ converges to $Y^{*}, w$ to $w^{*}$, and $\hat{k}$ to $\hat{k}^{*}$. If, however, another specification of expectations formations were made, there is nothing to rule out the possibility of cyclical oscillations.

\section{Vintage Models and Econometric Estimation}

Over the past decade, three of the focal points of econometric research have been the estimation of the residual ${ }^{26}$ (that part of the growth of output that cannot be explained by the growth in factor inputs), of the elasticity of substitution, and of the investment function. Most of this work has employed an aggregate production function with malleable capital. If in fact capital is not malleable, then a specification error has been made; it is the purpose of this section to explore the implications of this specification error. ${ }^{27}$

${ }^{26}$ See, for instance, Solow (1957), Denison (1962), and Griliches and Jorgenson (1967).

${ }^{27}$ In this section only, all real variables are measured extensively, that is, in natural units, rather than intensively, that is in labor units. 


\section{The Residual}

Solow (1957) in his classic paper assumed a production model which would have the form if there were no technological change,

$$
Y=F(K, L) \text {. }
$$

By differentiating the logarithm of (7.1), and assuming that factors were paid their marginal products, he obtained,

$$
\frac{\dot{Y}}{Y}=(1-\alpha) n+\alpha \frac{\dot{K}}{K} \quad \text { where } \quad 1-\alpha=\text { share of labor } .
$$

The difference between the actual increase in output and the right-hand side of the above equation is the residual.

For our vintage model, again under the assumption of no technological change, and also for simplicity, under the assumption of no depreciation $(\mu=0),{ }^{28}$ we obtain

$$
\frac{\dot{Y}}{Y}=(1-\alpha) n+I \frac{r}{Y}
$$

where $r=[f(\hat{k})-w] / \hat{k}$ is the instantaneous rate of return (the quasi-rents on the type of machines being constructed at the moment).

The first question that arises is, are these two models ever identical? They are, if and only if for some valuation of the capital stock, $\hat{K}$, we have $\alpha=$ $r \hat{K} / Y$. But this is equivalent to $r \hat{K}=\Pi$, where

$$
\Pi=\int_{\mathbf{k}}^{\infty}[f(k)-w] d N(k, t)=Y-w,
$$

or $\hat{K}=\Pi / r$, which is the value of the capital stock if wages and the rate of return were to remain constant. This valuation of the capital stock is equal to the market valuation of the capital stock if and only if the economy has static expectations. Moreover, when $w$ and $r$ are changing, this definition has the obvious defect that $\dot{\hat{K}} \neq I$.

The second question that arises is, what kind of residual would one obtain if one used the market value of the capital stock, $V$, where

$$
V(t)=\int_{0}^{\infty} q(k, t) k d N(k, t) ?
$$

Since all capital goods must have the same rate of return, that is,

$$
\frac{\dot{q}(k, t)}{q(k, t)}=r(t)-\frac{f(k)-w(t)}{q(k, t) k} \text { for all } k,
$$

${ }^{28}$ We should mention that the inclusion of depreciation $\mu>0$ adds another source of bias in that the right-hand side of (7.3) then becomes $(1-\alpha) n+\alpha \mu+$ $I(r / Y)$. Thus, for example, even when capital is measured so that $I(r / Y)=\alpha(I / K)$ (see the discussion directly following), the residual includes a depreciation component (in fact a component just equal to $\alpha \mu$ ). 
we obtain

$$
\int_{0}^{\infty} \dot{q}(k, t) k d N(k, t)=r(t) \int_{0}^{\infty} q(k, t) k d N(k, t)-\int_{0}^{\infty}[f(k)-w(t)] d N(k, t)
$$

or Capital Gains $=r(t) V(t)-\Pi(t)$. Hence we obtain

$$
\frac{\dot{Y}}{Y}=(1-\alpha) n+\alpha \frac{I}{V}+\frac{\text { Capital Gains }}{Y} \frac{I}{V} .
$$

Thus, even if there were no technological changes, by using market valuation of the capital stock, one would obtain a residual of (Capital Gains/ $Y$ ) $\times(I / V)$. Again, it should be noticed that $I \neq \dot{V}$.

A third approach to the problem is to consider each of the different types of capital goods as a separate factor. For example, assume that there are a finite number of different types of capital goods. Then denote by $\alpha_{k}$ the share in national income of profits on machines of type $k$, that is, if employment on machines of type $k$ is denoted by $d N(k, t)$, then

$$
\alpha_{k}=\frac{f(k)-w}{Y} d N(k, t) \text {. }
$$

It immediately follows that

$$
\frac{\dot{Y}}{Y}=\left(1-\sum_{k} \alpha_{k}\right) n+\alpha_{\hat{k}} \frac{I}{V(\hat{k})},
$$

where $V(\hat{k})$ is the value (and number, since $q[\hat{k}, t]=1$ ) of machines of type $\hat{k}$,

$$
V(\hat{k})=\hat{k} d N(\hat{k}, t) .
$$

Observe that $\alpha_{\hat{k}}$ is always less than the share of profits, but $V(\hat{k})$ is less than the total value of the capital stock.

\section{Elasticity of Substitution}

Since the Arrow, Chenery, Minhas, and Solow paper (1961), numerous attempts, using both cross-section and time-series data, have been made to estimate the elasticity of substitution. Almost universally, these have assumed a constant elasticity of substitution, malleable capital model of the form (here specialized to include only Harrod neutral technical progress).

$$
Y=\left\{\delta K^{-\rho}+(1-\delta)[L A(t)]^{-\rho}\right\}^{-(1 / \rho)},
$$

so that

$$
\ln w=(\rho+1)[\ln (Y / L)-\ln A(t)]
$$


First, it is important to observe that if one did a cross-section or timeseries estimate of the elasticity of substitution based on the assumption of malleability, the resulting estimate would have (except if the economies were in steady-state balanced growth) nothing to say about the elasticity of substitution of the ex ante production function if capital were not malleable.

The following calculations suggest, moreover, why (at least in the context of time-series data) one might obtain an estimate of the elasticity less than unity for a developing economy. Taking the time derivative for the above equation, we obtain,

$$
\frac{\dot{w}}{w}=(1+\rho)\left(\frac{\dot{Y}}{Y}-n\right)-(1+\rho) \frac{\dot{A}}{A},
$$

whereas in our model we obtain

$$
\frac{\dot{w}}{w}=(1+\gamma)\left(\frac{\dot{Y}}{Y}-n\right)-(1+\gamma) \delta
$$

where

$$
(1+\gamma)^{-1}=\frac{\beta w}{N}\left(1-\frac{w N}{Y}\right), \delta=\frac{s}{\hat{k}}\left(f(\hat{k})-\frac{Y}{N}\right)
$$

and

$$
\beta=\frac{\partial N(\mathbf{k}, t) / \partial \mathbf{k}}{f^{\prime}(\mathbf{k})} .
$$

For a developing economy, the output per man on new machines is greater than average output per man. Hence $\delta>0$. But for economies near balanced growth, $[\partial N(\mathbf{k}, t) / \partial \mathbf{k}] / N$ decreases approximately exponentially; so $(1+\gamma)$ becomes arbitrarily large. In finite time $(1+\gamma)>1$, so the "apparent" elasticity is less than unity, and for paths very near balanced growth, much less than unity.

\section{Investment Behavior}

In the models considered thus far, employment is exogenous and output endogenous. An alternative view is to consider output as determining employment. (This is, of course, the Keynesian approach.) On the basis of this interpretation of the model, a theory of investment may be derived, which can be compared to the neoclassical theories of investment which assume malleable capital (see Jorgenson [1963]; Bischoff [1968]). Consider the simplified form of the theory as exposited by Jorgenson, where the malleable capital production function is Cobb-Douglas with capital exponent $\alpha$. (In order to make the comparison with our model as simple as possible, we will ignore all lags.) If $r$ is the rate of return on capital, $r=\partial F / \partial K=$ $\alpha Y / K^{D}$, where $K^{D}$ is the desired capital stock. In the absence of lags, desired and actual capital stock are equal; investment is just equal to the 
difference between capital stock today and desired capital stock tomorrow, plus replacement, that is,

$$
I=\frac{\alpha \dot{Y}}{r}-\alpha \frac{Y}{r} \frac{\dot{r}}{r}+\frac{\alpha \mu Y}{r}
$$

where $\mu$ is the rate of depreciation.

This should be compared with the equivalent result for our model, where, if the unemployment rate $u$ does not change,

$$
I=\frac{\dot{Y}}{r}+[-(1-u)(1-\alpha) \lambda+\mu] \frac{Y}{r},
$$

where, as usual, $r=[f(\hat{k})-w] / \hat{k}$, the quasi-rents on machines constructed today, and $\alpha$ is the share of capital. The important point to observe is that investment is affected by the level of the rate of interest but not (directly) by changes in the rate of interest. Moreover, the coefficient of the first term in our model is unity, independent of the ex ante production function, whereas in the neoclassical model it is the share of capital. (Of course, if one uses an incorrect measure of $r$-in particular, too small a value of $r$-it will result, if one estimates the equation unrestricted, in a coefficient of $\dot{Y} / r$ less than unity.) Finally, note that the coefficient, $Y / r$ is to be interpreted not as the rate of depreciation times the share of capital but as $\mu-(1-\alpha) \lambda^{29}$

Thus, if coefficients really are fixed ex post, equation (7.16) may represent a serious specification error. The next question that arises is, what is the correct value of $r$ to use in equation (7.17)? This depends on the nature of the expectations formation. Consider the two extremes: perfect foresight and static expectations. If individuals have perfect foresight, then $r$ equals profits plus capital gains divided by the value of the capital stock. ${ }^{30}$ If individuals have static expectations, the correct measure of $r$ is profits not including capital gains divided by the market value of the capital stock. ${ }^{31}$

It should be emphasized that these results are only suggestive of the kinds of errors that appear in econometric estimation when it is assumed that capital is malleable and in part it is not.

\section{Summary}

In this paper we have analyzed, under a number of alternative saving and expectations hypotheses, the dynamics of an economy in which once a machine is constructed its production characteristics are fixed. Although in

${ }^{29}$ If there are variations in the unemployment rate, $u$, equation (7.17) becomes $I=(\dot{Y} / r)+[\mu-(1-u)(1-\alpha) \lambda](Y / r)+u[(1-\alpha) Y / r]$.

${ }^{30}$ This is the measure correctly used by Bischoff (1968). Unfortunately, in other aspects, his model is not consistent with the perfect foresight assumption.

${ }^{31}$ Note that if and only if individuals have static expectations will the marginal capital-labor ratio $\hat{k}=f^{\prime-1}(r)$. 
the long run these economies look very much like the simple neoclassical models analyzed, for instance, by Solow and Swan, in that under all the alternative assumptions the economy converges to balanced growth, this model admits of a much richer variety of behavior along the path to steady growth. For instance, in the model with static expectations where saving depends on income distribution, there are likely to be capital intensityincome distribution cycles (although they are always damped). Under the assumption that a constant proportion of income is saved, whether there is static expectations or perfect foresight, income per capita may "overshoot" its long-run value-but only once; the wage rate, on the other hand, may increase or decrease several times, depending on the inherited capital stock. Moreover, in all cases, machines may be constructed which will eventually no longer be used because of economic obsolescence.

As in other models of accumulation with heterogeneous capital goods and perfect foresight, although the price system is unstable in the sense that if the initial prices of different kinds of capital goods are incorrect, then the system does not converge to balanced growth, such paths eventually violate one of the perfect foresight conditions and are not true equilibrium paths.

Optimal saving behavior, unlike that in the malleable capital model, results in roughly two phases of development: an earlier phase in which output is specialized in investment, and a later phase in which investment is specialized in a single technique. Both phases, as in the optimal evolution of the malleable capital model, result in providing production possibilities corresponding to the planner's rate of time preference.

Finally, because outside of balanced growth the quantitative as well as qualitative behavior of the economy is different from that of the malleable capital model, estimations of the residual, the elasticity of substitution, or investment functions based on the malleable capital model may be seriously misleading.

\section{References}

Akerlof, G. A. "Stability, Marginal Products, Putty and Clay," in K. Shell (ed.). Essays on the Theory of Optimal Economic Growth. Mass. Inst. of Tech. Press, 1967.

Arrow, K. J., Chenery, H. B., Minhas, B., and Solow, R. M. "Capital-Labor Substitution and Economic Efficiency," Rev. Econ. and Statis. (August, 1961), pp. 225-50.

Bischoff, C. "A Study of Distributed Lags and Business Fixed Investment." Unpublished Ph.D. dissertation, Mass. Inst. of Tech., 1968.

Calvo, G. "A Vintage Model of Optimum Economic Growth." Yale University, 1967 (mimeographed).

Champernowne, D. G. "The Production Function and the Theory of Capital: Comment,” Rev. Econ. Studies, XXI (1953-54), 112-35. 
Denison, E. F. The Sources of Economic Growth in the United States and the Alternatives Before Us. New York: Committee for Economic Development, 1962.

Fisher, F. M. "Embodied Technical Change and the Existence of an Aggregate Capital Stock," Rev. Econ. Studies (October, 1965), pp. 263-68.

Griliches, Z., and Jorgenson, D. W. "The Explanation of Productivity Change," Rev. Econ. Studies (July, 1967), pp. 249-83.

Hahn, F. H. "Equilibrium Dynamics with Heterogeneous Capital Goods," Q.J.E. (November, 1966), pp. 633-46.

Johansen, L. "Substitution v. Fixed Production Coefficients in the Theory of Economic Growth: A Synthesis," Econometrica (April, 1959), pp. 157-76.

Jorgenson, D. W. "Capital Theory and Investment Behavior," A.E.R., Proc. (May, 1963), pp. 247-59.

Koopmans, T. C. "On the Concept of Optimal Economic Growth," in Semaine d'etude sur le role de l'analyse econometrique dans la formulation de plans de developpement. Pontifical Academy of Sciences, 1965.

Phelps, E. S. "Substitution, Fixed Proportions, Growth and Distribution," Internat. Econ. Rev. (September, 1963), pp. 265-88.

Ramsey, F. P. “A Mathematical Theory of Saving," Econ. J. (September, 1928), pp. 543-59.

Shell, K., and Stiglitz, J. E. "The Allocation of Investment in a Dynamic Economy," Q.J.E., (November, 1967), pp. 592-609.

Sheshinski, E. "Balanced Growth and Stability in the Johansen Vintage Model," Rev. Econ. Studies (April, 1967), pp. 239-48.

Solow, R. M. "A Contribution to the Theory of Economic Growth," Q.J.E. (February, 1956), pp. 65-94.

- "Technical Change and the Aggregate Production Function," Rev. Econ. and Statis. (August, 1957), pp. 312-20.

- "Substitution and Fixed Proportions in the Theory of Capital," Rev. Econ. Studies (June, 1962), pp. 207-18.

Solow, R. M., Tobin, J., von Weizsäcker, C. C., and Yaari, M. "Neoclassical Growth with Fixed Proportions," Rev. Econ. Studies (April, 1966), pp. 79-115.

Stiglifz, J. E. "A Note on Technical Choice under Full Employment in a Socialist Economy," Econ. J. (September, 1968), pp. 603-9.

Swan, T. W. "Economic Growth and Capital Accumulation," Econ. Rec. (November, 1956), pp. 334-61.

Tobin, J. "Liquidity Preference as Behavior Towards Risk," Rev. Econ. Studies (February, 1958), pp. 65-86.

Von Weizsäcker, C. C. "Existence of Optimal Programs of Accumulation for an Infinite Time Horizon," Rev. Econ. Studıes (April, 1965), pp. 85-104. 